\title{
IRF5 genetic risk variants drive myeloid- specific IRF5 hyperactivation and presymptomatic SLE
}

\author{
Dan Li, ${ }^{1}$ Bharati Matta, ${ }^{1}$ Su Song, ${ }^{1}$ Victoria Nelson, ${ }^{1}$ Kirsten Diggins, ${ }^{2}$ Kim R. Simpfendorfer, ${ }^{3}$ \\ Peter K. Gregersen, ${ }^{3}$ Peter Linsley, ${ }^{2}$ and Betsy J. Barnes ${ }^{1,4}$ \\ ${ }^{1}$ Center for Autoimmune, Musculoskeletal and Hematopoietic Diseases, The Feinstein Institutes for Medical Research, \\ Manhasset, New York, USA. ${ }^{2}$ Systems Immunology Division, Benaroya Research Institute at Virginia Mason, Seattle, \\ Washington, USA. ${ }^{3}$ Robert S. Boas Center for Genomics and Human Genetics, The Feinstein Institutes for Medical \\ Research, Manhasset, New York, USA. ${ }^{4}$ Departments of Molecular Medicine and Pediatrics, Zucker School of Medicine at \\ Hofstra/Northwell, Hempstead, New York, USA.
}

Genetic variants within or near the interferon regulatory factor 5 (IRF5) locus associate with systemic lupus erythematosus (SLE) across ancestral groups. The major IRF5-SLE risk haplotype is common across populations, yet immune functions for the risk haplotype are undefined. We characterized the global immune phenotype of healthy donors homozygous for the major risk and nonrisk haplotypes and identified cell lineage-specific alterations that mimic presymptomatic SLE. Contrary to previous studies in B lymphoblastoid cell lines and SLE immune cells, IRF5 genetic variants had little effect on IRF5 protein levels in healthy donors. Instead, we detected basal IRF5 hyperactivation in the myeloid compartment of risk donors that drives the SLE immune phenotype. Risk donors were anti-nuclear antibody positive with anti-Ro and -MPO specificity, had increased circulating plasma cells and plasmacytoid dendritic cells, and had enhanced spontaneous NETosis. The IRF5-SLE immune phenotype was conserved over time and probed mechanistically by ex vivo coculture, indicating that risk neutrophils are drivers of the global immune phenotype. RNA-Seq of risk neutrophils revealed increased IRF5 transcript expression, IFN pathway enrichment, and decreased expression of ROS pathway genes. Altogether, the data support that individuals carrying the IRF5-SLE risk haplotype are more susceptible to environmental/stochastic influences that trigger chronic immune activation, predisposing to the development of clinical SLE.

Authorship note: DL and BM contributed equally to this paper.

Conflict of interest: The authors have declared that no conflict of interest exists.

Copyright: (c) 2020, American Society for Clinical Investigation.

Submitted: August 6, 2018 Accepted: December 18, 2019 Published: January 30, 2020.

Reference information: JCI Insight. 2020;5(2):e124020. https://doi.org/10.1172/jci. insight.124020.

\section{Introduction}

Systemic lupus erythematosus (SLE) is a multifactorial autoimmune disease characterized by breakdown of tolerance to nuclear antigens, immune complex deposition, and multi-organ involvement. Circulating anti-dsDNA antibodies and antibodies against small nuclear RNA-binding proteins (anti-Ro, anti-LA, antiSM, and anti-RNP) are hallmarks of SLE (1-3). B cell hyperactivity also plays a central role in SLE. Higher numbers and frequency of circulating antibody-secreting cells (ASCs, plasma cells) associate with SLE disease activity and anti-dsDNA titer $(4,5)$. Patients with SLE display both innate and adaptive immune alterations, as well as granulocyte alterations that affect blood cell composition. Indeed, peripheral blood gene expression profiling identified an interferon (IFN) gene signature, a neutrophil-specific signature, and a plasma cell signature as biomarkers for SLE that correlate with disease activity (6-9).

With the success in genetic discoveries, an important challenge has been to define the mechanisms through which identified genes contribute to disease pathogenesis. The transcription factor IFN regulatory factor 5 (IRF5) is one such gene identified as an autoimmune susceptibility gene (10). IRF5 is a critical downstream mediator of MyD88-dependent Toll-like receptor (TLR) signaling that regulates both arms of the immune system - innate and adaptive (10-13). Genetic variants within or near IRF5 are robustly associated with SLE (14-18). To date, 4 IRF5 variants have been prioritized as candidate causal that strongly associate with SLE risk and make up the major risk haplotype in European Caucasians (Figure 1A and refs. 14-18). Two single nucleotide polymorphisms (SNPs) are in the $5^{\prime}$ and $3^{\prime}$ untranslated regions (UTRs) of IRF5 (rs2004640 and rs10954213, respectively). rs2004640 is reported to create an alternative splice site (16). 
rs10954213 alters the polyA signal,7 leading to expression of a shorter, more stable IRF5 mRNA $(17,19)$. rs10488631 is $5 \mathrm{~kb}$ downstream of IRF5 and its function still unknown (12). A 5-bp CGGGG insertion (rs142738614/rs77571059) in the 5' UTR of IRF5 was identified and found to create an additional binding site for Sp1 $(15,18)$. Given their locations in the regulatory regions of IRF5, it was postulated that IRF5 risk variants would lead to elevated $I R F 5$ expression. Indeed, others and we reported that $I R F 5$ risk variants generally correlated with elevated IRF5 expression in SLE blood cells and with IFN- $\alpha$ activity in patients with SLE positive for anti-RNA binding protein or anti-dsDNA antibodies (20-22). It has been difficult, however, to distinguish a genetic contribution from a nongenetic (disease-associated) one in patients with SLE because IFN- $\alpha$ itself transcriptionally upregulates IRF5 (23) and circulating SLE triggers, such as TLR-stimulating antigens, induce IRF5 activation and nuclear translocation $(11,12,24,25)$. It is thus conceivable that previous findings of IRF5 expression and activation in SLE blood cells was due to disease-associated factors (IFNs and TLR ligands) rather than genetic contributions (25). As such, the immune phenotype driven by IRF5 genetic risk in healthy donors is currently undefined, and whether, or how, genetic risk triggers alterations in specific cell lineages rather than globally is not known.

Here, we demonstrate that different genetic backgrounds in healthy donors profoundly influence the immune phenotype in a cell type-specific manner. In particular, we characterized alterations in the blood of healthy donors who are homozygous for either the major IRF5 risk haplotype or the common nonrisk haplotype. Our data support the notion that integrated functional analysis of cells derived from genetically defined healthy donors may uncover the origin of predisposition to immune cell dysfunction, which in turn may lead to autoimmune diseases, such as SLE.

\section{Results}

The IRF5 homozygous risk haplotype confers elevated anti-nuclear antibody and anti-Ro positivity. Conditional logistical regression of disease-associated alleles in the IRF5 locus reveals that 3 variant groups within the IRF5 risk haplotype are independently associated with SLE (17). This suggests a complex mechanism of association and the possibility of more than 1 causative allele. To simplify the analysis for functional association studies, we selected 2 haplotypes of IRF5 for comparison (Figure 1A). The homozygous nonrisk $(\mathrm{B} / \mathrm{B})$ and risk $(\mathrm{E} / \mathrm{E})$ haplotypes contain the complete set of protective and risk alleles, respectively, of the primary candidate causal SLE-associated variants (Figure 1A).

We obtained fresh human blood from healthy donors who have no personal or family history of autoimmune or inflammatory disease from the GaP Registry (26) to assess an IRF5 risk phenotype over time. Multiple independent blood draws were obtained over a 4-year period for immune phenotyping. It was previously shown that patients with SLE, before clinical diagnosis, have elevated anti-nuclear antibody (ANA) staining (1). By ANA-HEp2, we found that healthy donors who are homozygous for the IRF5 risk haplotype were ANA positive; sera from SLE patients with clinically high and low dsDNA titers were used as positive controls (Figure 1, B and C). We tested for dsDNA antibodies by ELISA and were somewhat surprised to find no difference in the levels between risk and nonrisk individuals, while serum from SLE controls showed the expected high and low titers (Figure 1D). We then used an autoantibody array to examine other candidate antibodies considered pathogenic in patients with SLE and found that healthy donor risk carriers had a specific and significant increase in anti-Ro (SS-A; $60 \mathrm{kDa}$ ) antibodies (Figure 1, E-H). The observed increase in anti-Ro antibodies is reminiscent of findings from Cherian et al. in which they reported that asymptomatic, healthy individuals carrying an IRF5 risk haplotype were anti-Ro positive and at risk for progression to SLE (27). These data suggest that the IRF5 risk haplotype may initiate or drive the production of select autoantibodies before clinical disease onset.

IRF5 homozygous risk carriers have increased circulating plasma cells. Elevated ASCs have commonly been detected in the circulation of patients with $\operatorname{SLE}(4,5)$. By flow cytometry, we detected a significant increase in $\mathrm{CD}_{4} 5^{+} \mathrm{CD} 19^{+} \mathrm{IgD}^{-} \mathrm{CD} 38^{+}$plasma cells in the blood of homozygous risk versus nonrisk donors (Figure 2, A and B). This effect was subset specific because percentages of naive, transitional, nonswitched, and switched memory B cells were similar between donor groups (Supplemental Figure 1; supplemental material available online with this article; https://doi.org/10.1172/jci.insight.124020DS1). Importantly, fresh blood was obtained for plasma cell analysis from at least 3 independent blood draws per donor over a 4-year period. The observed increase in circulating plasma cell numbers from risk donors was conserved over time (Supplemental Figure 1E). To address whether the homozygous risk haplotype was required for the observed phenotype, we obtained fresh blood from healthy donors carrying varied combinations of 
A

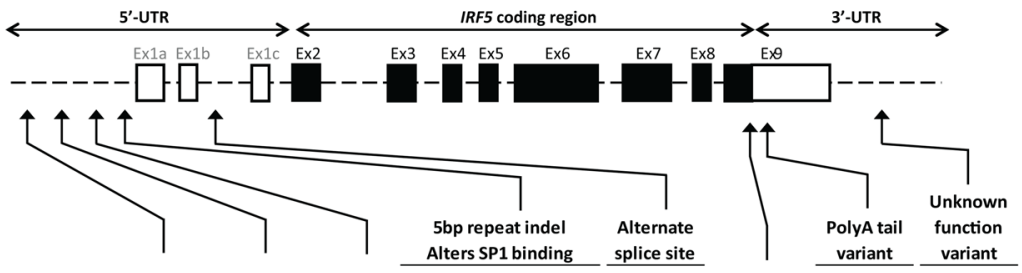

Group /

Haplotype Frequency rs729302 rs4728142 rs3757385 rs 142738614 rs 77571059 rs 2004640

\begin{tabular}{|rr|c|c|c|c|c|c|c|c|}
\hline A & 0.335 & A & A & G & GCGGG & T & T & A & T \\
\hline Non-risk B & 0.156 & C & G & T & - & G & T & G & T \\
\hline C & 0.148 & A & G & T & - & G & T & G & T \\
D & 0.099 & C & G & G & - & G & T & A & T \\
\hline Risk E & 0.085 & A & A & G & GCGGG & T & C & A & C \\
\hline F & 0.057 & A & G & G & - & T & T & G & T \\
G & 0.050 & C & G & T & - & G & T & A & T \\
H & 0.018 & A & G & G & - & T & T & A & T \\
I & 0.015 & C & A & G & GCGGG & T & T & A & T \\
J & 0.011 & A & G & G & GCGGG & T & C & A & C \\
\hline
\end{tabular}

$\begin{array}{lllllllllll}\text { Chr } 7 \text { position hg19 } 128568960 & 128573967 & 128577304 & 128577914 & 128577932 & 128578301 & 128589000 & 128589427 & 128594183\end{array}$ GaP subject selection

\section{C}
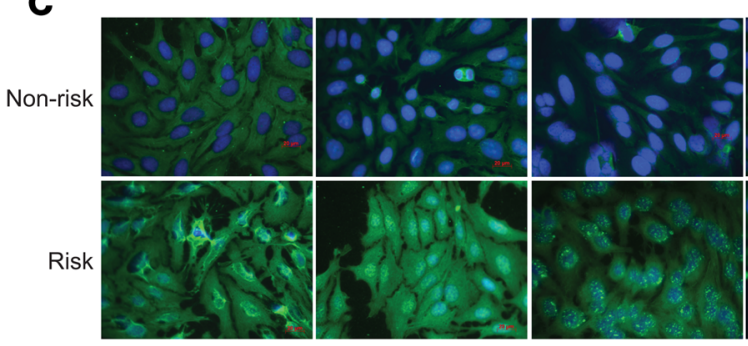

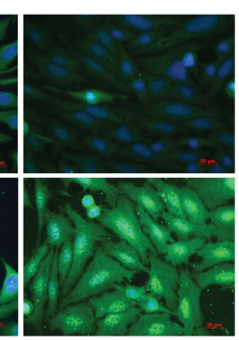

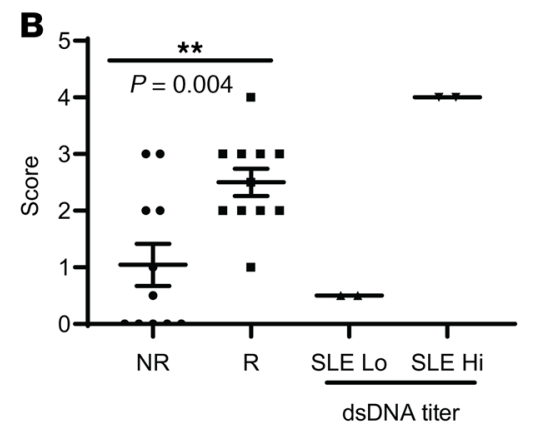

D

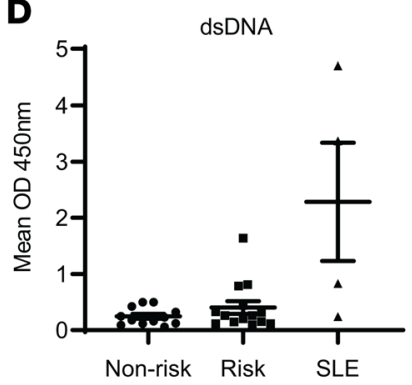

$\mathbf{E}$

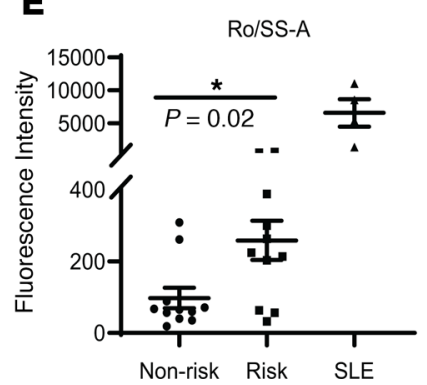

$\mathbf{F}$

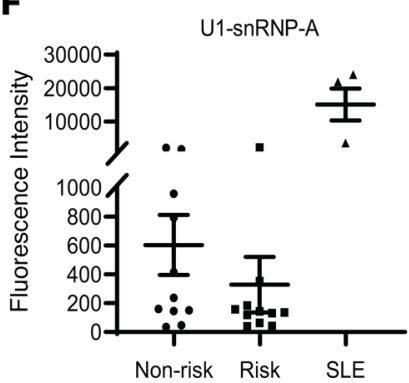

G

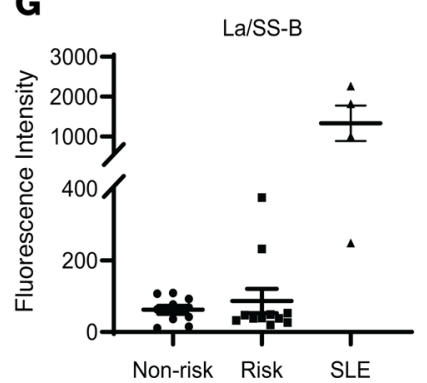

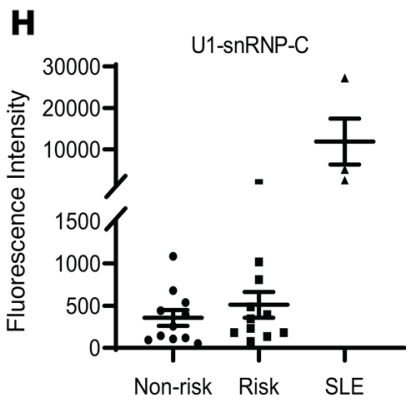

Figure 1. Healthy donors carrying the homozygous IRF5-SLE risk haplotype are ANA and anti-Ro positive. (A) Candidate causal SNPs associated with SLE are shown relative to the human IRF5 gene. IRF5 haplotypes were built in Caucasian subjects from the 1000 Genomes Project (74). Variants selected for inclusion in the haplotypes were candidate causal or associated with SLE in CWAS and thus proxies for the candidate causal variants. Genotype and Phenotype (GaP) Registry subjects were selected based on the indicated immunochip SNPs as homozygous for the nonrisk haplotype (B/B), homozygous for the risk haplotype (E/E), or other combinations of the IRF5 haplotypes. (B) ANA immunofluorescence scoring for C, including positive (dsDNA ${ }^{\text {hi }}$ ) and negative (dsDNA ${ }^{10}$ ) control SLE serum ( $n=4 ; 1: 500$ dilution); sera from $n=11$ risk and nonrisk donors. Zero represents a negative signal; 4 represents the strongest signal (Mann-Whitney $U$ test; comparisons are between risk and nonrisk healthy donors). (C) Representative ANA images from homozygous nonrisk $(n=5)$ and risk donors $(n=5)$ are shown with a serum dilution of 1:2 at original magnification $\times 200$. (D) Anti-dsDNA Ig concentrations were determined by ELISA with a 1:5 dilution of GaP serum from nonrisk (NR) and risk (R) donors and 1:20 dilution of SLE serum (unpaired 2-tailed $t$ test between nonrisk and risk donors). (E-H) Anti-Ro/SS-A (TROVE2) (E), anti-U1-snRNP-A (SNRPA) (F), anti-La/SS-B (SSB) (C), and anti-U1-snRNP-C (SNRNPC) (H) concentrations were determined by Luminex assay with a 1:5 serum dilution for GaP Registry donors and 1:20 for SLE donors (unpaired 2 -tailed $t$ test between nonrisk and risk donors). Single data points represent individual donors; sera from $n=11$ risk and nonrisk donors. Plotted data are after background subtraction. Data are presented as mean or mean $\pm \mathrm{SEM}$. ${ }^{*} P \leq 0.05{ }^{* *} P \leq 0.01$. Experiments in B-H were done twice.

IRF5 risk variants at the locus (Figure 1A). Quite strikingly, we found that the observed increase in plasma cell numbers was detected only in healthy donor homozygous risk carriers (E/E) and not those carrying other haplotypes (Figure 2C). Last, we addressed whether increased plasma cells seen in homozygous risk donors were due to an intrinsic defect in plasma cell differentiation. We recently reported that IRF5 plays 
A

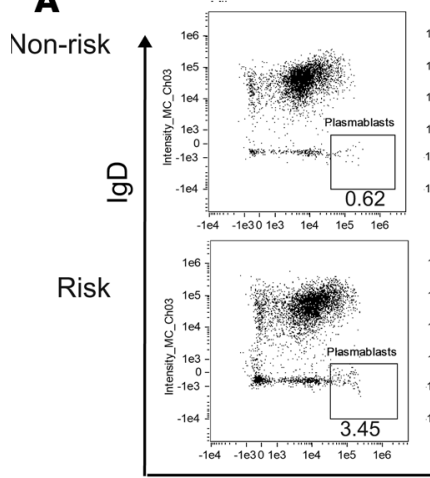

B

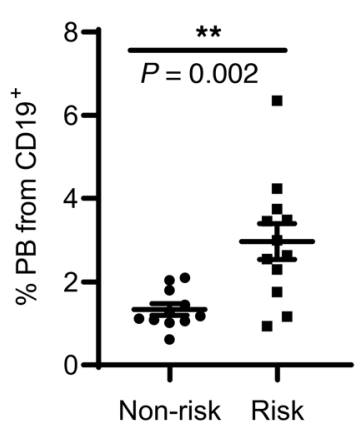

D
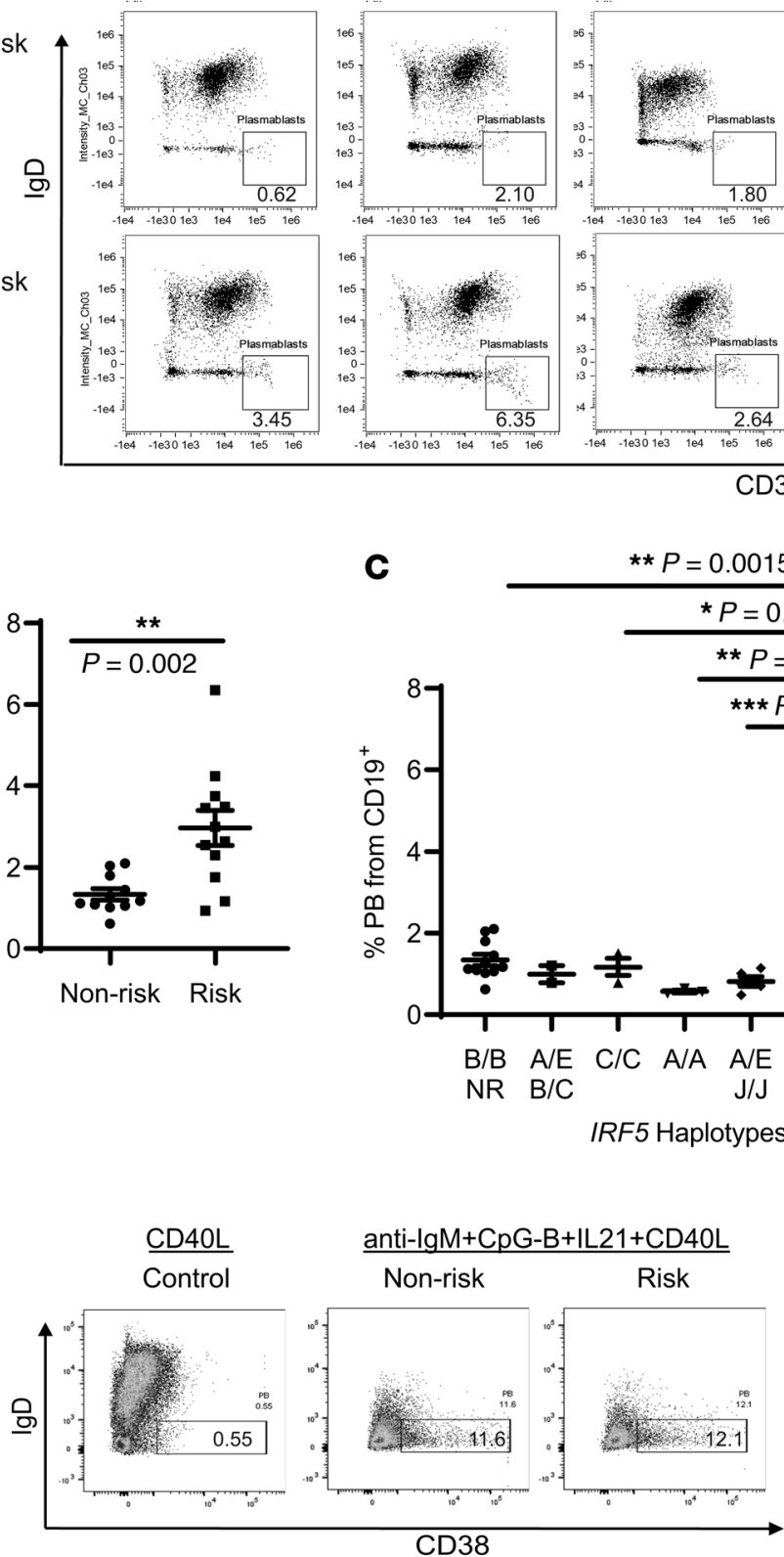

C
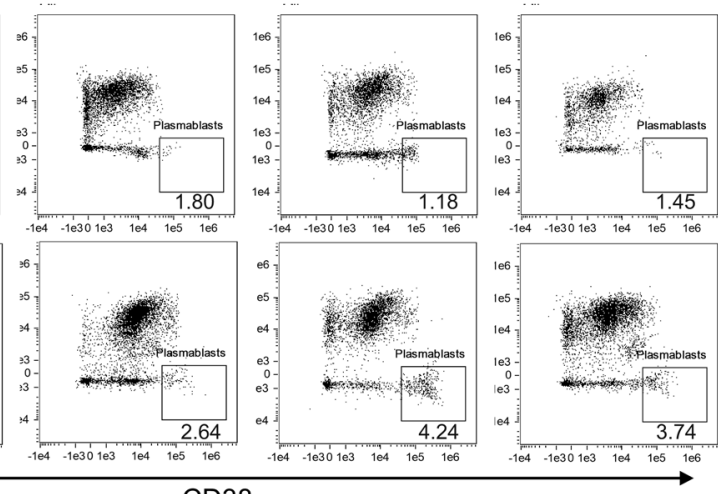

CD38
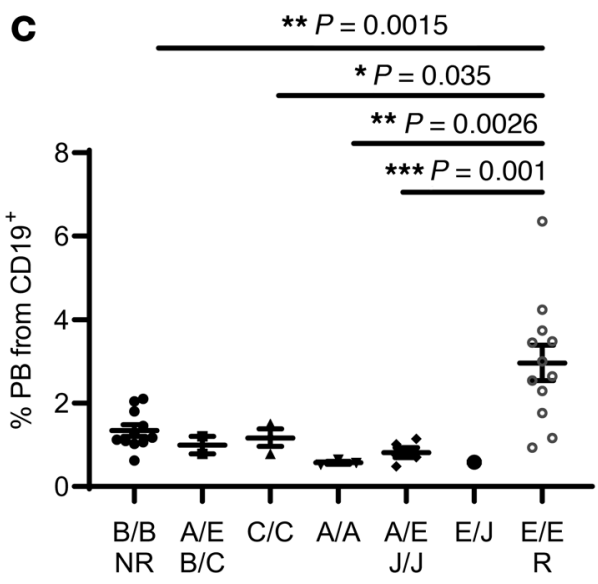

IRF5 Haplotypes

\section{E}

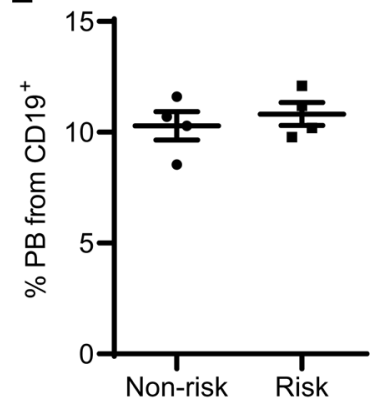

Figure 2. Plasma cells are elevated in the circulation of IRF5 homozygous risk donors. (A-C) Freshly isolated PBMCs were surface-stained and plasma cells gated as $C D 45^{+} C D 19{ }^{+} I g D^{-} C D 38^{+}$(PB, plasmablasts). (A) Representative dot plots from flow cytometry are shown from a single round of blood draws from independent homozygous risk $(n=5)$ and nonrisk $(n=5)$ donors. A is pregated for CD45 and CD19. (B) The number of circulating PBs from $n=$ 12 risk and $n=11$ nonrisk donors is shown as a percentage of the CD19+ gate (unpaired 2-tailed $t$ test). (C) Similar to B except percentage of PBs from homozygous nonrisk, risk, and representative mixed haplotypes from Figure 1A are plotted together (1-way ANOVA with Bonferroni's multiple-comparisons test using risk [E/E] as control group and excluding groups $A / E B / C$ and $E / J$ because of insufficient sample size. (D) Isolated naive $B$ cells from homozygous nonrisk and risk donors were in vitro cultured for 7 days with $150 \mathrm{ng} / \mathrm{mL}$ CD $40 \mathrm{~L}$ alone or with $100 \mathrm{ng} / \mathrm{mL} \mathrm{IL}-21$, $10 \mu \mathrm{g} / \mathrm{mL}$ anti-lgM antibody, and $2.5 \mu \mathrm{g} / \mathrm{mL} \mathrm{CpG}-\mathrm{B}$ to drive PB differentiation. Representative dot plots from flow cytometry analysis of a single matched nonrisk and risk donor after 7 days of culture. (E) Summarized data from D of $n=4$ risk and nonrisk donors are shown (unpaired 2-tailed $t$ test). Experiments were repeated 4 or more times (A and $\mathbf{B})$, were performed once (C), or were repeated twice ( $\mathbf{D}$ and $\mathbf{E})$. Single data points represent individual donors. Data are presented as mean \pm SEM. ; ${ }^{*} P \leq 0.05 ;{ }^{* *} P \leq 0.01 ;{ }^{* * *} P \leq 0.001$. 
an important role in TLR9/B cell receptor-induced ASC differentiation from human primary naive B cells (13). Purified naive B cells from homozygous risk and nonrisk donors were cultured in the presence of CD40L alone or CD40L, anti-IgM antibody, CpG-B, and IL-21 for 7 days to generate plasma cells (13). Somewhat surprisingly, we did not detect a difference in the generation of plasma cells between donor groups; both yielded similar numbers after 7 days in vitro culture (Figure 2, D and E). These data indicate that the observed difference in numbers of circulating ASCs is not due to intrinsic B cell differences, nor is it likely due to a single IRF5 genetic variant.

The IRF5 homozygous risk haplotype has little effect on IRF5 expression. Current dogma suggests that IRF5 risk variants contribute to SLE disease pathogenesis by increasing IRF5 expression levels (14-22, 27, 28). Previous findings from our lab indicated that IRF5 expression was elevated in monocytes, B cells, and dendritic cells (DCs) from patients with SLE compared with healthy controls (20). To determine whether the observed increase in circulating plasma cells was due to alterations in IRF5 expression within B cell subsets from risk and nonrisk donors, we used flow cytometry to assess intracellular IRF5 protein expression. Contrary to our expectations, we did not detect a significant difference in IRF5 expression levels in any B cell subset examined between risk and nonrisk donors (Figure 3, A and B), nor could we detect significant differences in any other cell type examined (monocytes, plasmacytoid dendritic cells [pDCs], neutrophils) (Figure 3, C-F, and Supplemental Figure 2, A-C). Of note, we detected a trend toward increased IRF5 expression in all monocyte subsets from risk donors that was not significant. Further, no difference in IRF5 expression after stimulation with the TLR7 ligand R848 was detected between donor groups (Supplemental Figure 2, D-L).

IRF5 homozygous risk carriers have increased basal and TLR-induced IRF5 activation in myeloid cells. We next examined whether IRF5 genetic risk contributes to basal IRF5 activation potential. We have previously shown that IRF5 activation is increased in monocytes from patients with SLE, and patients with SLE carrying the IRF5 homozygous risk haplotype express a distinct profile of IRF5 alternatively spliced transcripts with discrete cellular localization $(23,24,29)$. Using imaging flow cytometry, we assessed basal and TLR7-induced IRF5 cellular localization in immune cells from homozygous risk and nonrisk donors (Supplemental Figure 3 and ref. 24). We initially focused on B cell subsets, given the observed phenotype of elevated ASCs and ANA staining in risk donors; however, no difference in IRF5 activation levels was seen (Figure 3, G and H). We next examined IRF5 activation in other cell types that may drive ASC differentiation and antibody production, such as monocytes, pDCs, and neutrophils (30). We found a significant increase in basal IRF5 activation in total $\mathrm{CD} 14^{+}$monocytes from risk donors (Figure 3I). After subset gating, a significant increase in basal IRF5 activation, which we refer to as IRF5 hyperactivation, was found in every monocyte subset $\left(\mathrm{CD} 14^{+} \mathrm{CD} 16^{\text {lo }}\right.$ classical, $\mathrm{CD} 14^{\mathrm{lo}} \mathrm{CD} 16^{+}$inflammatory, and $\mathrm{CD} 14^{+} \mathrm{CD} 16^{+}$intermediate) analyzed (Figure 3J). Analysis of IRF5 hyperactivation in pDCs and neutrophils from risk and nonrisk donors gave similar results (Figure 3, K and L). Of note, IRF5 hyperactivation was further elevated in monocytes and pDCs after stimulation with R848 (Figure 3, M and N). Because pDC-produced IFN- $\alpha$ is a known driver of ASC differentiation $(31,32)$, we examined levels of intracellular IFN- $\alpha$. Although we were unable to detect basal IFN- $\alpha$, a significant increase in the percentage of IFN- $\alpha^{+}$pDCs was found in risk donors after R848 stimulation (Figure 3O). Combined, these data support 2 important findings: (a) direct effects of the IRF5 risk haplotype on IRF5 are seen only in the myeloid compartment, and (b) monocytes, $\mathrm{pDCs}$, and neutrophils from risk donors are already primed for IRF5 activation.

Elevated levels of circulating pDCs in homozygous risk donors. The observed increase in plasma cell numbers, along with IRF5 hyperactivation in pDCs, monocytes, and neutrophils from risk donors, raises the question of lineage-specific genetic effects. Although we did not detect differences in the percentage of other B cell subsets, further analysis of myeloid subsets revealed a significant increase in the number of circulating pDCs (Figure 4, A and B). Distinct from pDCs, however, no significant change in the percentage of circulating monocytes (classical, intermediate, and inflammatory) was detected (Supplemental Figure 4, A and B). Although these data support lineage-specific effects of the IRF5 risk haplotype, they raise questions about the causative pathway that could be driving plasma cell differentiation and autoantibody formation because intrinsic B cell defects were not found in subjects carrying the IRF5 risk haplotype (Figure 2, D and E).

Elevated spontaneous NETosis in homozygous risk donors. In search of potential antigenic triggers that may be driving IRF5 hyperactivation, pDC expansion, IFN- $\alpha$ production, and ASC differentiation in risk donors, we turned our attention toward neutrophils because they make neutrophil extracellular traps (NETs) in response to antigen that ultimately expose the immune system to autoantigens (33). It was previously reported that neutrophils from patients with SLE are more prone to release NETs and that NETs 
activate pDCs to produce IFN- $\alpha(34,35)$. Immune complexes composed of neutrophil-derived peptides and self-DNA along with antibodies against these peptides and self-DNA were found in SLE serum (34). Although we did not detect a difference in IRF5 protein expression between neutrophils of risk and nonrisk donors (Figure 3F), we detected a significant increase in IRF5 hyperactivation (Figure 3L) and spontaneous NETosis (Figure 4, C-E). Under normal circumstances, NETs are cleared by degradation, but in patients with SLE, there appears to be an imbalance between NET formation and clearance $(36,37)$. By 3 independent methods, we examined NETosis in risk and nonrisk donors, as well as a few positive control patients with SLE. Isolated neutrophils were cultured for 4 hours and then stained with anti-CD66b antibodies and cit-H3 and MPO antibodies for flow cytometry analysis. Double-positive neutrophils demarcate those undergoing NETosis. Homozygous risk donors had significantly elevated levels of spontaneous NETosis, which were similar to positive-control SLE neutrophils, as compared with nonrisk donors (Figure 4, C and D). Alternatively, isolated neutrophils were plated on poly-D-lysine-coated coverslips and stained with either Sytox green and DAPI or cit-H3 and MPO. In addition to SLE neutrophils, PMA-induced NETs were used as a positive control (Figure 4E). Based on these data, we reanalyzed serum from a cohort of donors in Figure 1 for MPO anti-neutrophil antibodies and found that levels were significantly elevated in risk donors (Supplemental Figure 4C). Together, these data suggest that similar to neutrophils from subgroups of patients with SLE $(36,37)$, neutrophils from homozygous risk donors are more likely to produce NETs, which may serve as an antigenic source that drives autoimmunity.

Elevated IRF5 transcript expression and a type I IFN gene signature in risk neutrophils. To begin to understand why risk neutrophils undergo spontaneous NETosis, we first examined expression of one of the inducers of NETosis - neutrophil chemotactic factor IL-8 $(38,39)$. IL-8 is generally first expressed in monocytes/macrophages because they are often the first cells to respond to antigen, but IL- 8 can be secreted by most cells that express TLRs (40). We sorted inflammatory and classical monocytes from risk and nonrisk donors and performed real-time quantitative PCR (qPCR) for IL8 expression. We detected a significant ( $\sim 2$ - to 3-fold) increase in $I L 8$ expression in both monocyte subsets from risk donors (Supplemental Figure 4D). These data suggest that the observed increase in IRF5 hyperactivation in risk monocytes may contribute to elevated IL8 expression.

We also detected a significant, albeit small, increase in neutrophil IRF5 hyperactivation (Figure 3L), suggesting that IRF5 may be differentially driving neutrophil function. To examine this, freshly isolated neutrophils were obtained from a cohort of donors, and RNA was isolated for whole-transcriptome sequencing. Somewhat surprisingly, we detected a trend of increased IRF5 transcript expression in risk neutrophils (Figure 5A and Supplemental Table 1). These data were independently confirmed by qPCR in a larger cohort of donors, revealing an approximately 4-fold increase in IRF5 transcript expression in risk neutrophils (Figure 5B). Gene enrichment analysis revealed that risk neutrophils display an expression profile similar to type I IFN- and TLR-induced activation (35). We detected significant enrichment of the IRF7 and IFN- $\gamma$ gene signatures that included upregulated expression of NOD-like receptors, TLR signaling molecules, and ubiquitin ligases (Figure 5, C and D, and Supplemental Tables 1 and 2). Guanylate binding proteins (GBPs) were among the top expressed genes in both the IRF7 and IFN- $\gamma$ modules (Supplemental Tables 1 and 2). GBPs are a family of dynamin-related large GTPases that are expressed in response to IFNs and other proinflammatory cytokines to mediate a broad range of pathogen-induced innate immune responses (41). In immune cells, GBPs activate NLRP3 and AIM2 inflammasomes in conjunction with bacterial signals to induce pyroptosis (42-47). Together, these data suggest that another potential mechanism of enhanced NETosis in risk neutrophils is via IFN signaling because IFNs are reported to prime neutrophils for NETosis $(38,48-50)$.

Somewhat surprisingly, we also detected decreased expression of key players in the reactive oxygen species (ROS) pathway, such as glutathione peroxidases, superoxide dismutases, peroxiredoxin 6 , and glucose-6-phosphate dehydrogenase, that resulted in a significant reduction in ROS signaling in risk versus nonrisk neutrophils (Figure 5, C and D, and Supplemental Table 3). Alterations in the ROS pathway and/ or ROS production from neutrophils of patients with SLE have been implicated in SLE disease onset and progression (51). Although there is some discordance between findings, because both increased and decreased ROS production have been reported in SLE neutrophils (51-54), more recent data indicate that NADPH oxidase 2-mediated (NOX2-mediated) ROS deficiency increases the risk of developing autoimmune diseases (55-60). ROS-deficient mice and humans with mutations in neutrophil cytosolic factor 1 (NCF1), or other subunits of the NOX2 complex, were found to have a prominent type I IFN gene signature 

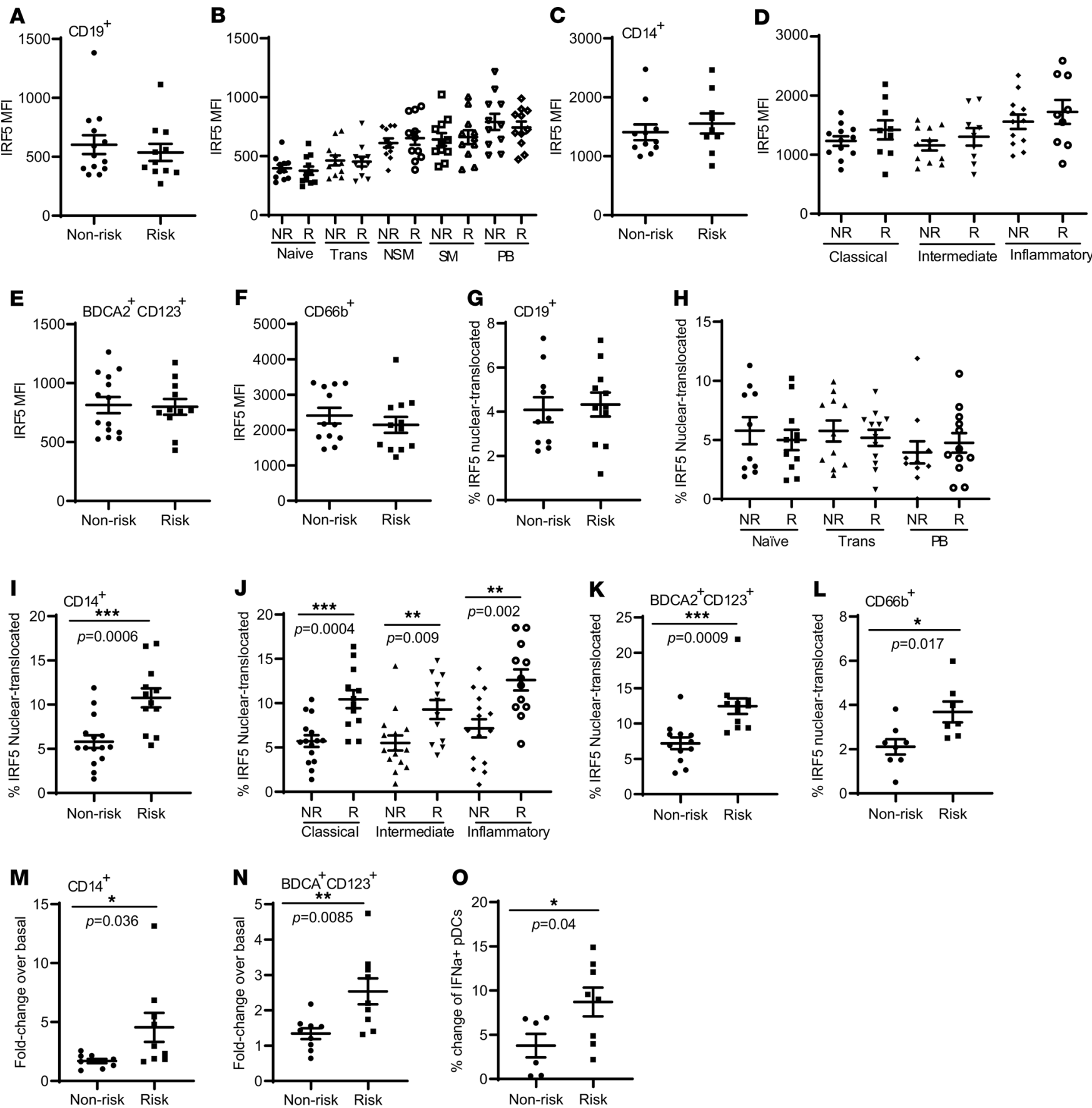

Figure 3. The IRF5-SLE risk haplotype has no effect on IRF5 expression but drives IRF5 hyperactivation in myeloid cells. (A and B) IRF5 expression was determined in B cell subsets from $n=11$ homozygous risk and nonrisk donors as mean fluorescence intensity (MFI) by flow cytometry. Naive, transitional (trans), nonswitched memory (NSM), switched memory (SM), and PBs were examined. (C-F) Monocyte subsets from $n=9$ homozygous risk and $n=12$ nonrisk donors (C and $\mathbf{D}), \mathrm{pDCs}$ from $n=10$ homozygous risk and $n=12$ nonrisk donors (E), and neutrophils from $n=$ 12 homozygous risk and nonrisk donors (F) were examined (unpaired 2-tailed $t$ test between NR and R for each group). (G-L) IRF5 activation was determined in same cell subsets as A-F by imaging flow cytometry. Percentage of IRF5 ${ }^{+}$cells within a given subset that contain nuclear-localized IRF5 is shown (unpaired 2-tailed $t$ test). (M) A further increase in IRF5 activation was seen after stimulation of PBMCs from nonrisk and risk donors with $500 \mathrm{ng} / \mathrm{mL}$ R848 for 2 hours. Fold change in percentage of IRF5 nuclear localization is shown after gating on IRF5+CD14 ${ }^{+}$monocytes in unstimulated (basal) and stimulated cells (unpaired 2-tailed $t$ test between NR and R for each group). (N) Same as $\mathbf{M}$ except fold change in IRF5 activation is shown in pDCs after R848 stimulation (unpaired 2-tailed $t$ test). (0) Percentage of pDCs from $\mathbf{N}$ that are positive for intracellular IFN- $\alpha$ is shown between nonrisk and risk donors after stimulation of PBMCs with R848 (unpaired 2-tailed $t$ test). Experiments were repeated 4 or more times (A, C, $\mathbf{E}, \mathbf{G}$, and $\mathbf{I}$ ), were repeated 3 times (B, D, J, K, $\mathbf{M}$, and $\mathbf{N}$ ), or were repeated twice (F, $\mathbf{H}, \mathbf{L}$, and $\mathbf{0})$. Single data points represent individual donors. Data are presented as mean $\pm \mathrm{SEM}$. ${ }^{*} P \leq 0.05 ;{ }^{* *} P \leq 0.01 ;{ }^{* *} P \leq 0.001$. 

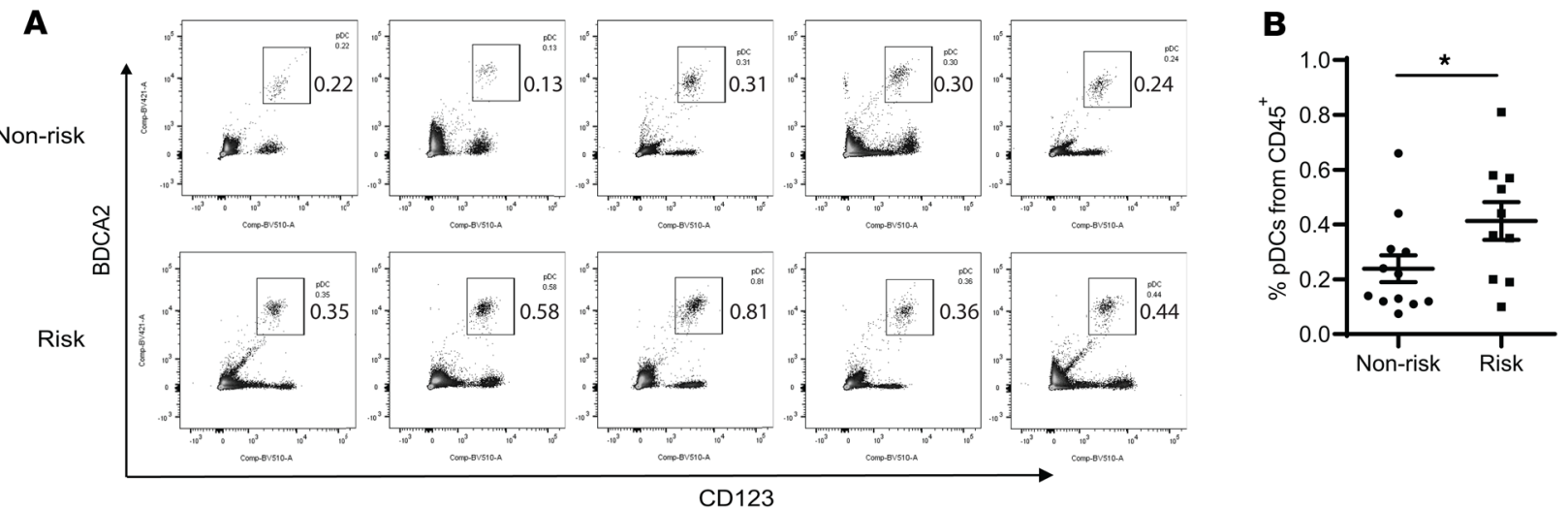

C
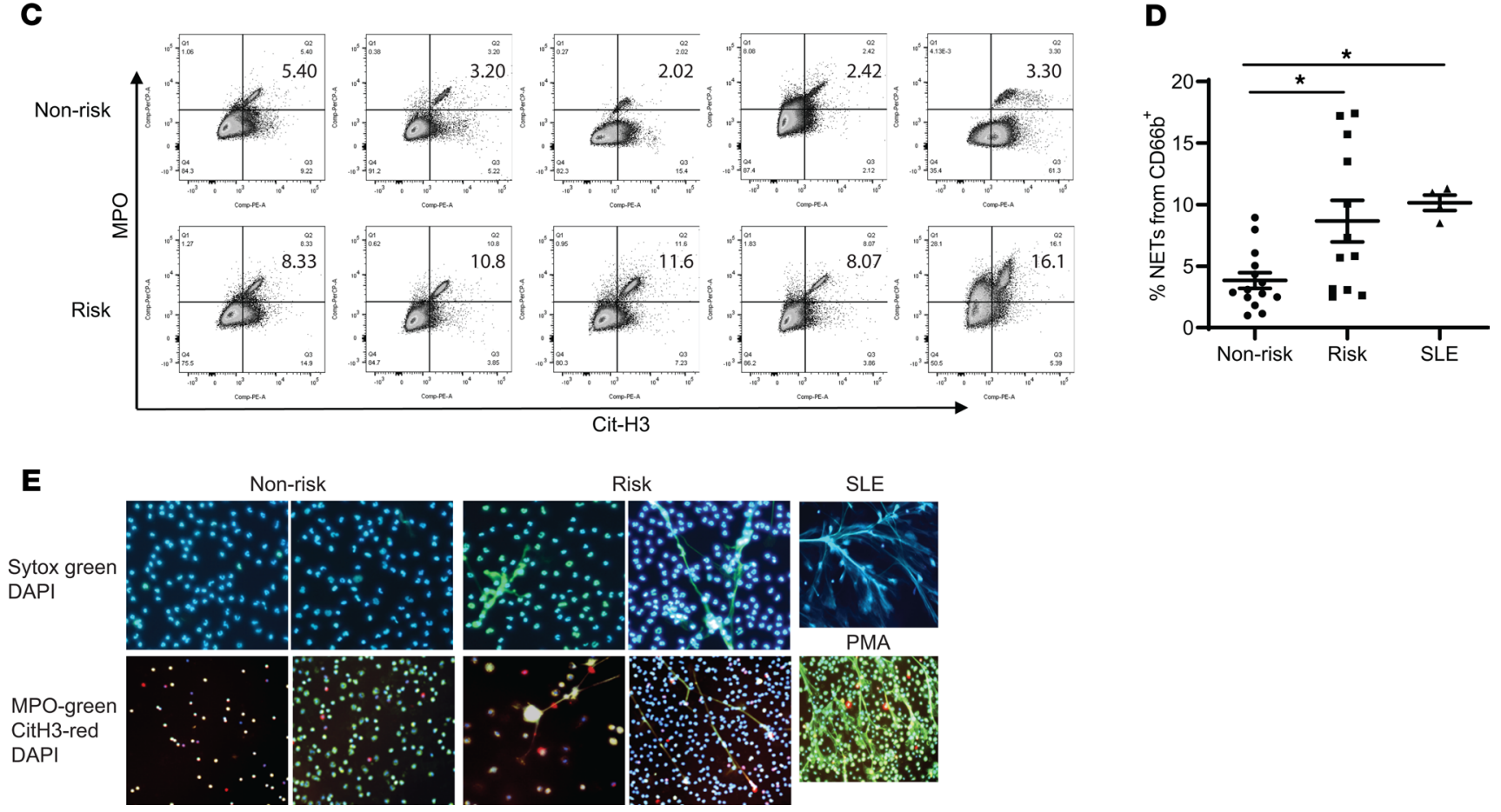

Figure 4. IRF5 homozygous risk donors have elevated numbers of circulating pDCs and spontaneous NETosis. (A and B) Similar to Figure 2, A and B, except freshly isolated PBMCs were surface-stained and pDCs gated as $C D 45^{+} C D 123^{+} B D C A 2^{+}$. (A) Representative dot plots from flow cytometry are shown from a single round of blood draws. A is pregated for CD45. (B) The number of circulating pDCs from $n=10$ risk and $n=12$ nonrisk donors is shown as a percentage of CD45 ${ }^{+}$gate

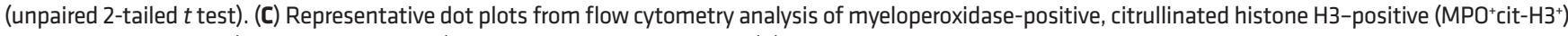
NETs in donor samples ( $n=5$ risk and nonrisk). C is pregated on CD66b cells. (D) Quantification of NETs from $n=12$ risk and $n=14$ nonrisk donors is shown as a percentage from CD66b+ cells (1-way ANOVA with Tukey's multiple-comparisons test). (E) The presence of NETs was visualized by plating equal numbers of freshly isolated neutrophils from homozygous nonrisk, risk, and patients with SLE on poly-L-lysine-coated coverslips for 4 hours. Representative images are from staining with Sytox green and DAPI or MPO, Cit- $\mathrm{H3}$, and DAPI. PMA was used as a positive control for NET induction on nonrisk neutrophils (original magnification $\times 20$ ). Experiments were repeated 3 times (A-E). Single data points represent individual donors. Data are presented as mean \pm SEM. ${ }^{*} P \leq 0.05$.

with elevated autoantibodies (58). Although we did not detect a difference in expression of Ncf1, it has previously been reported that expression levels do not necessarily correlate with function (59). Altogether, these data support that risk neutrophils have altered biology, including elevated IRF5 transcript expression, IRF5 hyperactivation, and enrichment of an IFN gene signature, that may lead to the presymptomatic autoimmune phenotype detected in risk donors.

Ex vivo coculture of healthy PBMCS with SLE neutrophils induces IRF5 activation in $p D C S$, pDC expansion, and ASC differentiation. We next sought to determine whether risk neutrophils (NETs) are an antigenic trigger that drives the $I R F 5$ risk immune phenotype. To do this, we developed an ex vivo coculture system using SLE neutrophils and healthy donor PBMCs. To control for HLA donor mismatch effects, cocultures consisted of (a) neutrophils (N) from healthy donor 1 plated with matched PBMCs (P) (H1-N + H1-P), (b) 
A

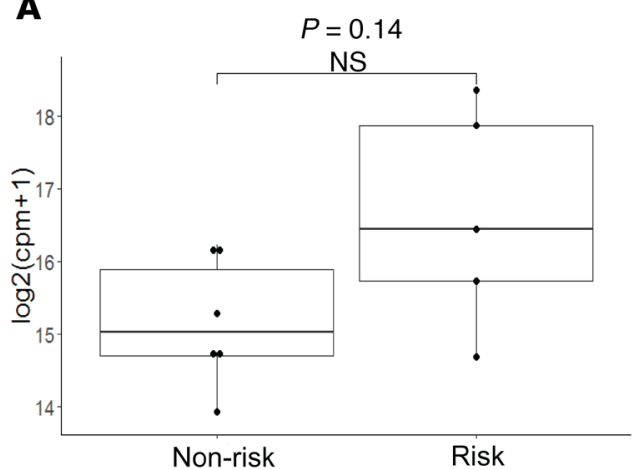

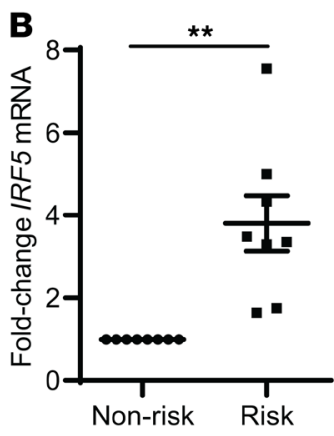

D
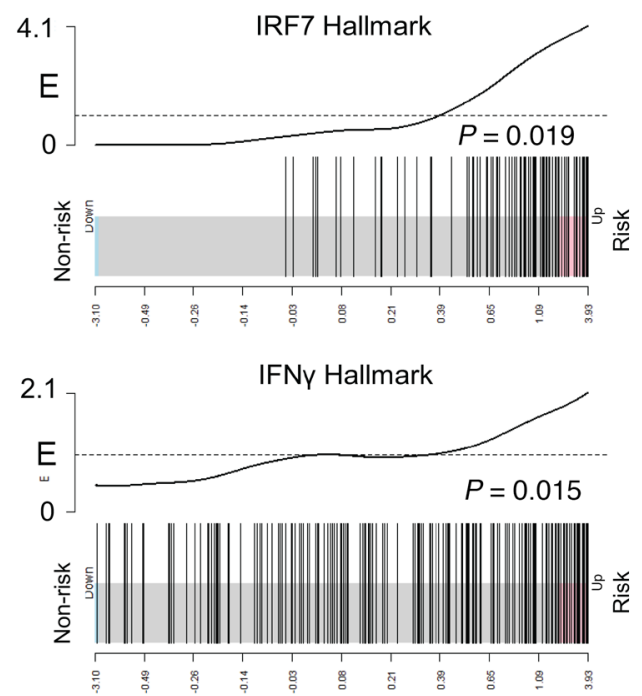

ROS Hallmark
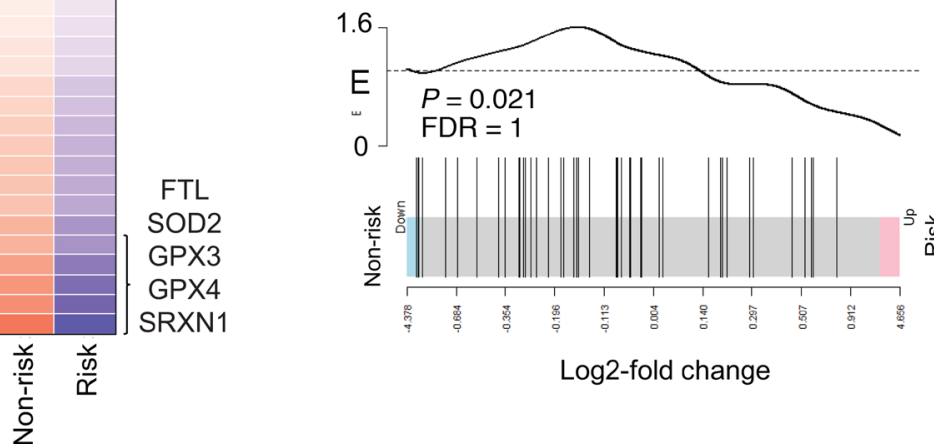

FTL

SOD2

GPX3

GPX4

SRXN1

GCEF1

PFKP

PRNP

PRDX2

Z-score

$-0.5$

$-0.5$

$\frac{x}{\frac{x}{x}}$

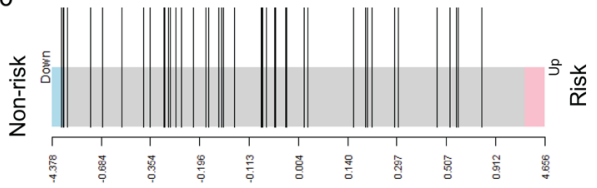

Log2-fold change
ROS

SOD2

RAPGEF6

CASP3

ISOC1
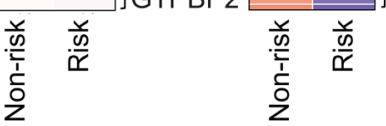

Figure 5. Risk neutrophils express elevated IRF5 transcript expression and are enriched for IRF7 and IFN- $\gamma$ gene signatures. (A) RNA-Seq analysis of IRF5 transcript expression in neutrophils from risk $(n=5)$ and nonrisk $(n=6)$ donors. Median $\log _{2}$ expression of IRF5 between groups is shown by box plot (Wilcoxon's test). The box plot depicts the minimum and maximum values (whiskers), the upper and lower quartiles, and the median. The length of the box represents the interquartile range. (B) Independent confirmation of IRF5 transcript expression in neutrophils from $n=8$ risk and nonrisk donors by qPCR analysis. Data are represented as fold change in mRNA expression in risk compared with nonrisk (unpaired 2-tailed $t$ test). (C) Heatmaps generated from RNA-Seq of risk and nonrisk neutrophils show the top 3 most enriched gene sets (IRF7/type I IFN, IFN- $\gamma$, and ROS). Relative gene expression was calculated as per-gene $Z$ scores across all samples, and the mean $Z$ score calculated for each gene per group is shown. (D) Neutrophil expression signatures of genes differentially expressed within each module/hallmark between risk and nonrisk donors are shown (limma's roast function). Error bars show mean \pm SEM. ${ }^{* *} P \leq 0.01$.

neutrophils from healthy donor 2 plated with healthy donor 1 PBMCs (H2-N + H1-P), and (c) neutrophils from patients with SLE plated with healthy donor 1 PBMCs (S-N + H1-P). Neutrophils were cultured for 4 hours to generate NETs (Figure 4E), followed by addition of PBMCs. Functional outcome was assessed at 3 time points. At 4 hours' coculture, IRF5 activation in pDCs was determined by imaging flow cytometry. 
After overnight coculture, $\mathrm{pDC}$ activation via $\mathrm{CD} 40^{+}$surface staining and percentage of $\mathrm{pDC}$ from $\mathrm{CD} 45^{+}$ cells was determined by flow cytometry. After 7 days of coculture (13), plasma cell generation was determined. Somewhat expectedly, given the antigenic nature of SLE NETs, we detected a significant increase in IRF5 activation in pDCs after coculture with SLE NETs and not healthy donor neutrophils/NETs (Figure 6A). Further, we found a significant increase in the percentage of pDCs after overnight coculture with SLE NETs, as well as a significant increase in pDC activation (Figure 6, B-E). Most strikingly, after 7 days of coculture, we found that SLE NETs drove plasma cell differentiation (Figure 6, F and G). Importantly, little effect was seen from HLA mismatch controls at any time point examined, supporting that the observed immune responses to SLE neutrophils were specific. These data support the use of an ex vivo coculture system to identify drivers of IRF5 genetic risk and to confirm and mechanistically interrogate the observed in vivo immune phenotypes.

NETs from homozygous risk donors drive ASC differentiation. Using the same ex vivo coculture system, we examined whether the increase in spontaneous NETosis seen in risk donors was the driver of IRF5 hyperactivation and plasma cell differentiation. The only modification to the system was the use of neutrophils from risk (R-N) and nonrisk (NR-N) donors, rather than SLE neutrophils, cocultured with risk (R-P) and nonrisk (NR-P) PBMCs. No significant change in IRF5 activation in pDCs was seen between NR-P alone and NR-N plus NR-P after 4 hours' coculture, while coculture of R-N plus NR-P provided a significant increase in IRF5 activation (Figure 7A). This effect was cell type specific because the observed IRF5 hyperactivation in monocyte subsets (Figure 3, I and J) could not be replicated by ex vivo coculture (Supplemental Figure 5, A-C). The select effects of risk NETs on nonrisk pDC expansion and activation via CD40 staining were also conserved (Figure 7, B-E). Most striking was the recapitulation of increased plasma cell numbers by risk NETs on nonrisk PBMCs (Figure 7, F and G). Control data from the reverse cocultures are shown in Supplemental Figure 5, D-I, which support the specific effect of risk neutrophils and their spontaneous NETosis as drivers of the IRF5-SLE risk immune phenotype.

\section{Discussion}

In this study, we uncovered significant new insight into the relationship between IRF5 genotype, IRF5 expression/activation, and SLE risk. Remarkably, we found in healthy donors that the homozygous IRF5-SLE risk haplotype conferred basal IRF5 hyperactivation in myeloid cells that drove an SLE immune phenotype in both myeloid and lymphoid lineages. The observed increase in basal IRF5 activation was independent of IRF5 protein expression levels because no significant difference was found between nonrisk and risk donors (Figure 3). This is distinct from findings in patients with SLE where others and we reported an association of elevated IRF5 transcript and protein expression with patients carrying IRF5 risk variants (14-22). To our knowledge, this is the first comprehensive report of basal IRF5 expression levels in multiple subsets of untouched immune cells from healthy donors carrying IRF5 genetic risk. A recent report by Calise et al. confirmed a lack of effect of IRF5 haplotypes on IRF5 mRNA expression in unstimulated or stimulated B cells and monocytes (61). Previous related studies of IRF5 expression were in monocyte-derived macrophages (MDMs) and monocyte-derived DCs from healthy donors genotyped for rs 2004640 alone or in combination with rs2280714 (further downstream of rs10488631), revealing elevated expression after stimulation with TLR- and NOD2-like ligands or MDM polarization; however, basal IRF5 mRNA expression levels were not analyzed $(62,63)$. Only upon RNA-Seq and qPCR analysis of neutrophils from risk and nonrisk donors were we able to detect a significant increase in IRF5 transcript levels that associated with the homozygous risk haplotype (Figure 5, A and B). It is not currently clear whether the elevated IRF5 transcript expression is from genetic risk or a result of IFN exposure because IFN gene enrichment was detected by RNA-Seq (Supplemental Table 1) and IFNs are known to upregulate IRF5 expression (23). However, IFNs do not induce IRF5 activation (24), and risk serum was unable to induce IRF5 hyperactivation in nonrisk PBMCs. Thus, in the current study, the only direct effect of the homozygous IRF5 risk haplotype on IRF5 itself was the finding of IRF5 hyperactivation in myeloid cells from risk donors, including monocytes, pDCs, and neutrophils (Figure 3, I-L). We previously reported that the homozygous IRF5 risk haplotype in SLE monocytes determined the expression and abundance ranking of IRF5 transcript variants (29). Multiple IRF5 transcript variants exist that encode for distinct isoforms with discrete cellular localization and cell type-specific expression (23). For instance, some isoforms lack the IRF5 nuclear export signal and retain 1 or both nuclear localization signals, resulting in constitutively nuclear-localized IRF5 (23, 29, 64, 65). Unfortunately, because of a lack of sufficient depth in sequencing neutrophils from risk and nonrisk donors, we were unable 
to determine genotype-driven IRF5 transcription (29). Additional sequencing of myeloid cells from genotyped healthy donors will be required to address whether the IRF5 risk haplotype is driving a transcriptional program of IRF5 hyperactivation.

Another possible driver of IRF5 hyperactivation is the presence of constitutive antigenic triggers in homozygous risk donors. Accumulation of ANA specificities is a hallmark of impending clinical disease, and serum from patients with SLE can induce IRF5 nuclear translocation (24); yet many ANA-positive individuals remain healthy, suggesting that additional immune dysregulation underlies SLE pathogenesis $(1,66)$. In addition to ANA positivity with anti-Ro/SSA specificity, we detected increased anti-MPO antibodies that correlated with enhanced frequency of neutrophils undergoing spontaneous NETosis in homozygous risk donors. In SLE, neutrophils die upon exposure to SLE-derived anti-RNP antibodies and release NETs containing endogenous DNA and RNA, as well as neutrophil proteins (MPO) that activate $\mathrm{pDC}$ to produce IFN- $\alpha(34,35)$. In addition to stimulating multiple cell types, IFN- $\alpha$ is pivotal in driving the maturation of B cells into plasma cells, as well as priming neutrophils to undergo NETosis $(31,38,48-50,67-72)$.

Using an ex vivo coculture system, we found that SLE NETs and risk NETs are antigenic triggers capable of recapitulating the IRF5 risk immune phenotype in nonrisk PBMCs, resulting in IRF5 hyperactivation in pDCs, pDC activation and expansion (49), and increased plasma cell generation. Of note, we were unable to recapitulate IRF5 hyperactivation in nonrisk monocytes by coculture (Supplemental Figure 5, A-C). These data provide additional support that IRF5 hyperactivation in risk monocytes is genotype driven rather than induced by a circulating trigger. This is in contrast to our finding of IRF5 hyperactivation in risk $\mathrm{pDCs}$ that could be replicated by in vitro coculture of risk neutrophils with nonrisk PBMCs (pDCs), indicating that IRF5 hyperactivation in risk pDCs is dependent on the presence of circulating antigenic triggers rather than driven by the risk haplotype. In addition to NETs, we tested the possibility that risk serum alone could be an antigenic trigger driving the global IRF5 risk immune phenotype. Somewhat surprisingly, we were unable to induce ASC differentiation by coculture of nonrisk PBMCs with risk serum or SLE serum in the absence of neutrophils. Further, only SLE serum and not risk serum was able to induce IRF5 nuclear translocation in monocytes and pDCs from nonrisk donors (ref. 24 and data not shown). These data support that NETosis, and/or risk neutrophils, are key cellular components driving the IRF5 risk immune phenotype. Indeed, a significant body of literature now exists supporting an important role(s) for neutrophils in the development and regulation of SLE (73). Although little is currently known of IRF5 expression and function in neutrophils, data presented herein suggest that IRF5 may have a functional role in neutrophils, given its high level of expression compared with other lymphoid and myeloid cell types (Supplemental Figure 2C).

Interestingly, we detected a significant increase in the number of circulating $\mathrm{pDC}$ in risk versus nonrisk donors (Figure 4, A and B) that is distinct from patients with SLE (74-77). This distinction may be due in part to the lack of immune cell characterization in patients before a diagnosis of SLE (preclinical SLE). During the early stages of immune activation by virus or pathogen, peripheral $\mathrm{pDC}$ numbers increase through their proliferative capacity, as well as by replenishment from bone marrow progenitors (78). In the context of chronic immune activation, peripheral pDC numbers decrease through multiple mechanisms that include shortened life span, reduced proliferation, and enhanced apoptosis and trafficking to secondary tissues $(69,79-81)$. A recent report by Macal et al. sheds further light on the complex mechanisms by which pDC numbers and function are regulated (78). They found that TLR7 and type I IFN signaling contribute to peripheral $\mathrm{pDC}$ proliferation and cytokine production during the early stages of viral infection; however, after chronic infection, type I IFN led to the reduced de novo generation of $\mathrm{pDCs}$ from bone marrow progenitors, whereas sustained TLR7 signaling led to reduced IFN production, resulting in a state of $\mathrm{pDC}$ exhaustion $(78,82)$. Based on these findings, along with the observation of TLR- and IFN-induced gene enrichment in risk neutrophils (Figure 5, C and D), it is tempting to speculate that the observed expansion of peripheral $\mathrm{pDC}$ in healthy donor risk carriers represents a very early stage of innate immune activation in which cells have been exposed to low levels of pathogen or antigen that sensitizes them to a second hit, i.e., TLR ligand, rather than inducing a state of $\mathrm{pDC}$ exhaustion. Indeed, stimulation of risk PBMCs with $\mathrm{R} 848$ resulted in a significant increase in $\mathrm{pDC}$-mediated IFN- $\alpha$ production as compared with nonrisk PBMCs (Figure 3O). Altogether, data support that healthy donors carrying the IRF5 risk haplotype are more susceptible to environmental/stochastic influences that trigger chronic immune activation, predisposing to the development of clinical SLE. 
A
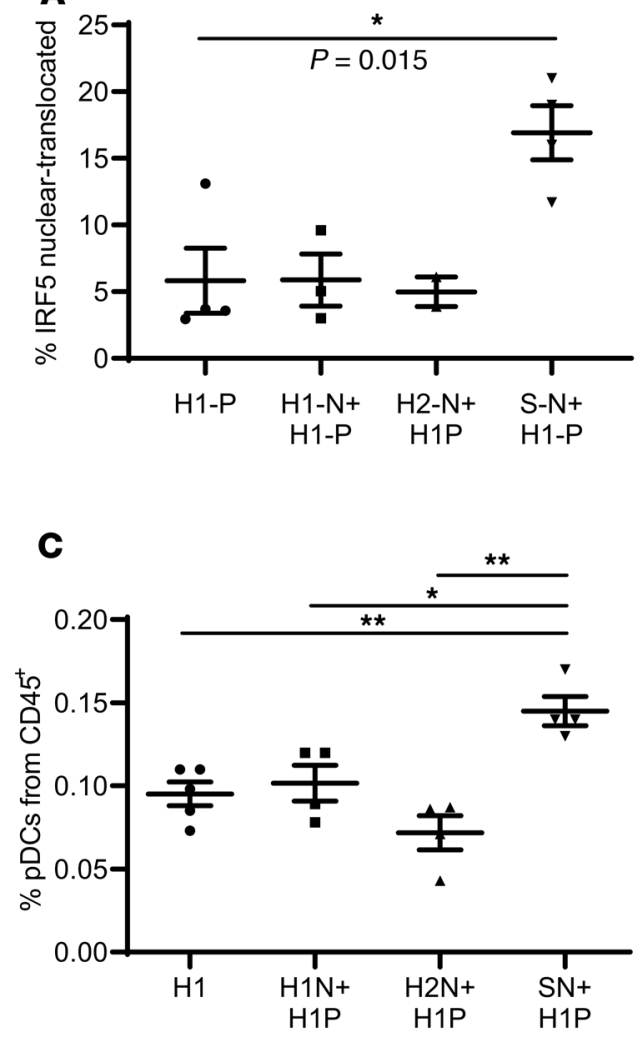

$\mathbf{E}$
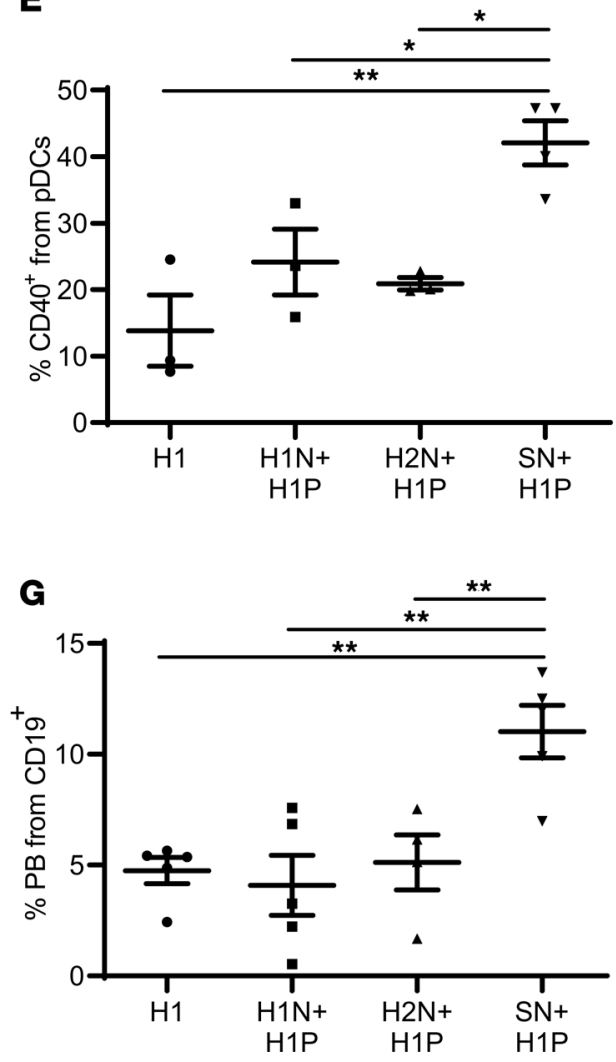

B

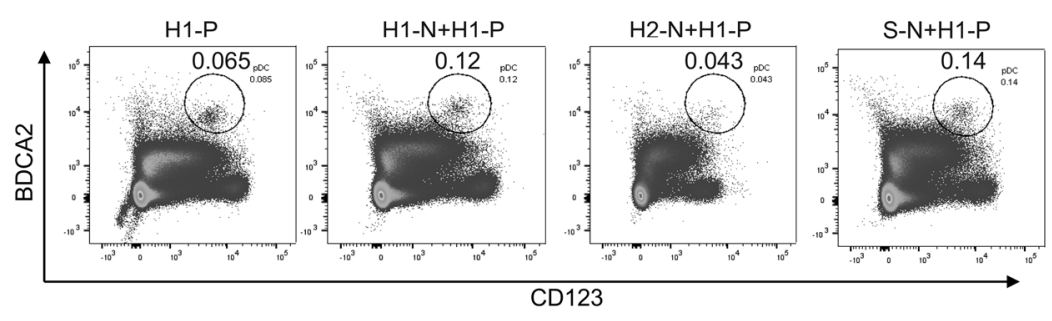

D

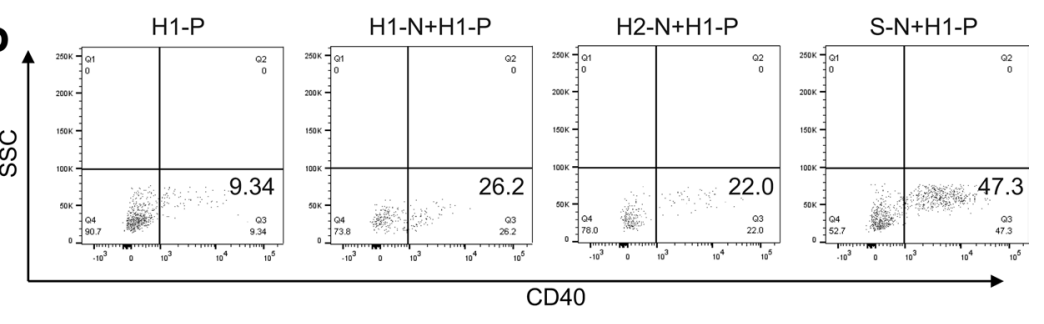

$\mathbf{F}$

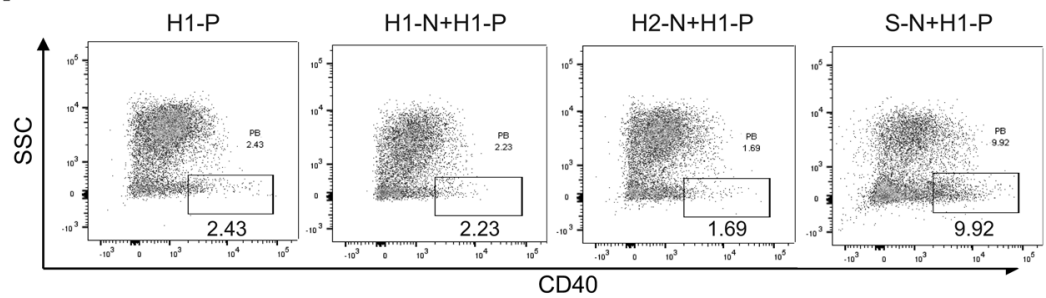

Figure 6. SLE NETs are an antigenic source that drives IRF5 activation in pDCs, pDC activation and expansion, and PB differentiation. (A-C) As in Figure 4E, neutrophils from patients with SLE (S-N) were isolated and plated on poly-L-lysine coverslips for 4 hours in a 24-well plate before addition of healthy donor PBMCs (H-P). Cocultures consisted of healthy donor 1 PBMCs (H1-P) plated alone, neutrophils (N) from healthy donor 1 plated with matched PBMCs ( $\mathrm{H} 1-\mathrm{N}+\mathrm{H} 1-\mathrm{P})$, neutrophils from healthy donor 2 plated with healthy donor 1 PBMCs ( $\mathrm{H} 2-\mathrm{N}+\mathrm{H} 1-\mathrm{P})$, and SLE neutrophils plated with healthy donor 1 PBMCs (S-N + H1-P). (A) Cells were harvested after 4 hours' coculture to examine IRF5 activation in pDCs by imaging flow cytometry (1-way ANOVA with Tukey's multiple-comparisons test; $n=4$ healthy and SLE donors). A second time point was harvested after overnight coculture to examine pDC numbers (B and $\mathbf{C}$ ) and activation via CD40 surface expression by flow cytometry ( $\mathbf{D}$ and $\mathbf{E}$ ) (1-way ANOVA with Tukey's multiple-comparisons test; $n=5$ healthy donors, $n=4 \mathrm{SLE}$ ). B is pregated on $\mathrm{CD} 45^{+}$cells and $\mathbf{D}$ on $\mathrm{CD} 45^{+} \mathrm{CD} 123^{+} \mathrm{BDCA2} 2^{+}$cells. A third time point was harvested after 7 days of coculture where PB differentiation was analyzed by flow cytometry (F). (G) Representative dot plots and summarized data are shown for the 4 conditions (1-way ANOVA with Tukey's multiple-comparisons test; $n=5$ healthy and SLE donor). Experiments were repeated 3 or more times. Single data points represent cells from individual donors. Data are presented as mean $\pm \mathrm{SEM}$. ${ }^{*} P \leq 0.05 ;{ }^{* *} P \leq 0.01$. 
A
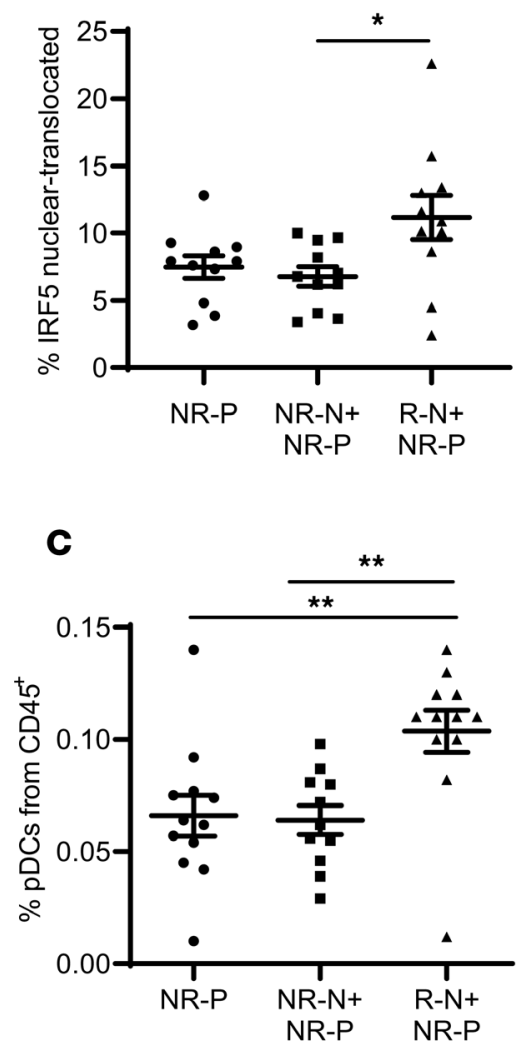

$\mathbf{E}$

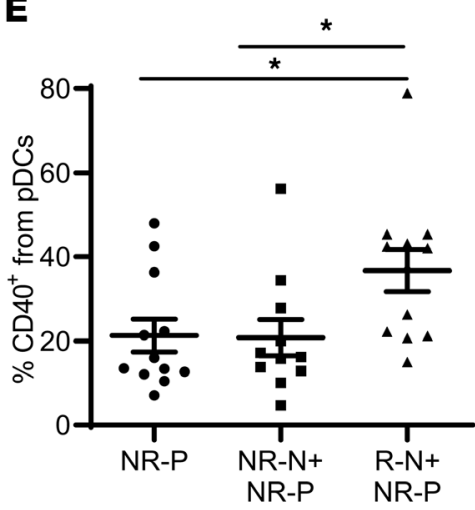

G

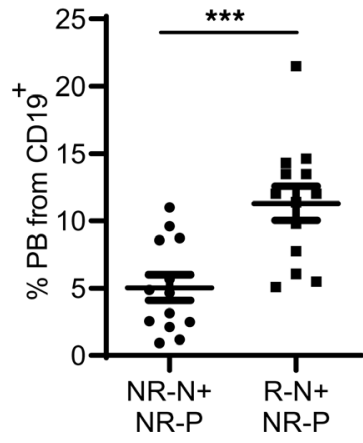

B
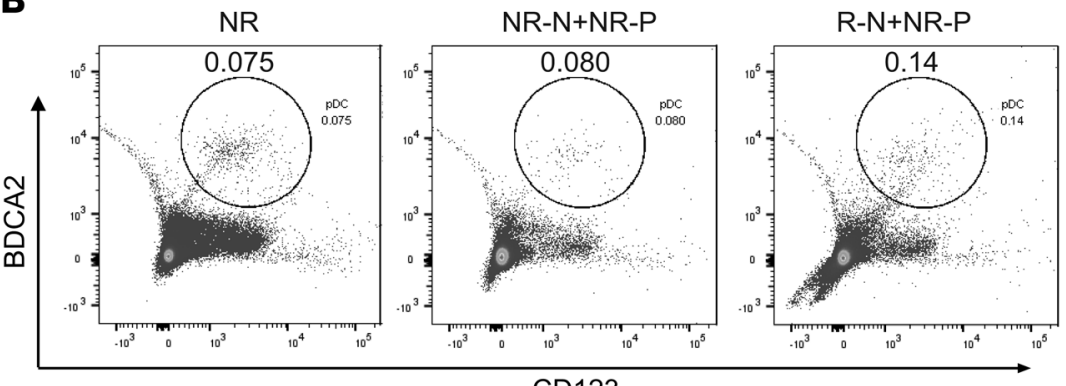

CD123

D
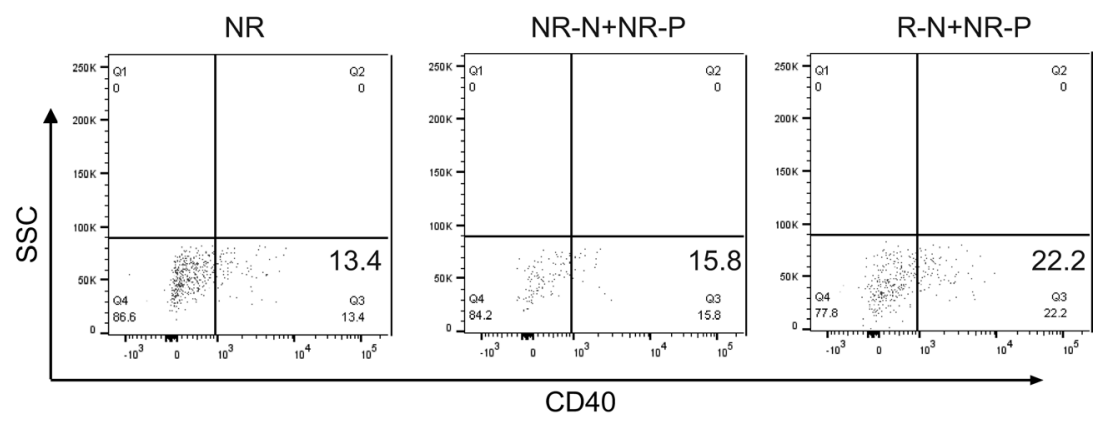

$\mathbf{F}$

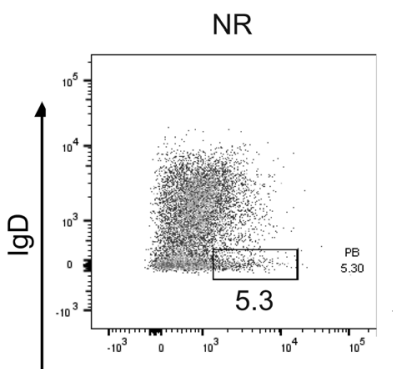

NR-N+NR-P

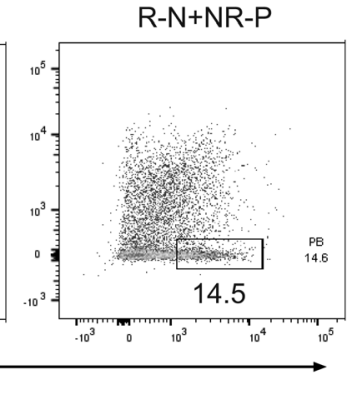

Figure 7. Ex vivo coculture of risk NETs with nonrisk PBMCs replicates the IRF5 risk immune phenotype. (A-G) Similar to Figure 6, neutrophils from nonrisk (NR-N) and risk donors (R-N) were plated and cocultured with nonrisk PBMCs (NR-P). PBMCs were harvested at the 3 time points for analysis. (A) Cells were harvested after 4 hours' coculture to examine IRF5 activation in pDCs by imaging flow cytometry (1-way ANOVA with Tukey's multiple-comparisons test; $n=11$ risk and nonrisk donors). (B) Cells were harvested after overnight coculture to examine $\mathrm{PDC}$ numbers (B and $\mathbf{C}$ ) and activation via CD40 surface expression by flow cytometry (D and E) (1-way ANOVA with Tukey's multiple-comparisons test; $n=12$ risk and nonrisk donors). $\mathbf{B}$ is pregated on $C D 45^{+}$cells and $\mathbf{D}$ on $C D 45^{+} \mathrm{CD} 123^{+} \mathrm{BDCA} 2^{+}$cells. (F) Cells were harvested after 7 days of coculture to examine PB differentiation by flow cytometry. Representative dot plots (F) and summarized data (G) are shown (unpaired 2-tailed $t$ test; $n=13$ risk and nonrisk donors). Experiments were repeated 3 times $(\mathbf{A}-\mathbf{G})$. Single data points represent cells from individual donors. Data are presented as mean \pm SEM. ${ }^{*} P \leq 0.05 ;{ }^{* *} P \leq 0.01 ;{ }^{* * *} P \leq 0.001$. 
Analogous to findings from Hedl et al. $(62,63)$, we detected an increase in TLR-induced cytokine production (IFN- $\alpha$ ) from risk cells that was likely due to constitutive IRF5 hyperactivation in pDCs (Figure $3, \mathrm{~N}$ and $\mathrm{O}$ ). These data are supported by the significant enrichment of genes involved in IFN- and TLR-induced activation in risk neutrophils (Supplemental Tables 1 and 2) that is reminiscent of gene pathways reported to be abundant in neutrophils of pediatric patients with SLE (49). In risk neutrophils, we also detected a significant reduction in the expression of genes involved in the ROS pathway (Supplemental Table 3). These data are intriguing because they provide additional support for the premise that reduced ROS drives autoimmune disease $(51,55-59)$; they also raise the question of a role for IRF5 in ROS production. Additional studies will be required to determine whether IRF5 is directly contributing to the ROS pathway. Altogether, data support that the IRF5-SLE risk haplotype confers genetic risk through myeloid cells, resulting in the expression of type I and II IFNs that drive NETosis and ASC differentiation, leading to elevated ANA. We propose that a certain "autoimmune" threshold must be reached for an asymptomatic, healthy donor IRF5-SLE risk carrier to convert to a symptomatic SLE patient.

\section{Methods}

GaP Registry donors and IRF5 haplotypes. Genotyped healthy donors used in the current study are participants of the GaP Registry (26). The GaP Registry is genotyped on the Illumina Human Immunochip, and genetic variants used for subject selection are shown in Figure 1A. In general, this study focused on male and female $\mathrm{GaP}$ donors who are either homozygous for the nonrisk haplotype (designated $\mathrm{B} / \mathrm{B}$ ) or homozygous risk (designated E/E) (Figure 1A). All donors are healthy with no personal or family history of autoimmune/inflammatory diseases or cancer. IRF5 haplotypes were built using LDlink (http://analysistools.nci.nih.gov/LDlink), which uses population genotype data from phase 3 of the 1000 Genomes Project (83). IRF5 haplotypes were generated in subjects of European ancestry (super population codes CEU, TSI, FIN, GBR, IBS), and similar haplotype frequencies were found in the GaP Registry. SNPs selected for inclusion in the haplotypes were candidate causal or proxies for candidate causal variants (14-22). Individual donors were called in a minimum of 4 times, unless otherwise indicated, over a 4 -year period for independent blood draws. Each single round of blood draws consisted of $n=11-13$ homozygous risk and $n=11-14$ homozygous nonrisk. Blood donation days were coordinated so that at least 1 risk and 1 nonrisk donor could give blood on the same day for comparative analysis. Serum and blood samples from $n=10$ patients with SLE (male and female) were obtained from the rheumatology clinic at Northwell Health. Each of the patients fulfilled at least 4 of the classification criteria for SLE as defined by the American College of Rheumatology.

Cell isolation. Buffy coats were prepared from fresh blood drawn by peripheral phlebotomy from genotyped healthy donors who participate in the GaP Registry (26). Blood was diluted 2-fold and subjected to Ficoll's density gradient separation (20). Isolated PBMCs were immediately used for experiments. Serum was isolated from undiluted blood and stored at $-80^{\circ} \mathrm{C}$ for later use. Granulocytes were purified from Ficoll's sediment by RBC lysis. The bottom layer of cells was collected after removing PBMCs. RBCs were lysed 2 times using RBC lysis buffer. The pellet was then washed once with PBS and resuspended in RPMI.

ANAs. Serum autoantibodies (GaP donors, 1:2 dilution in PBS; SLE donors, 1:500 dilution) were measured by ANA-HEp-2 (26104, Bio-Rad). Images were taken on a Zeiss Apotome microscope, at the same exposure time and magnification $(\times 200)$. Each image was read blindly and assigned an arbitrary score of 0-4; 0 represents a negative signal and 4 represents the strongest signal. Anti-dsDNA IgG was quantified by ELISA (Abnova). Serum autoantibodies were measured by multiplex detection with either the TruePLEX Antibody Profiling Array: Human Autoimmune I (AP100002, Origene, at a 1:5 dilution in PBS for GaP donors and 1:20 dilution for SLE donors) or the MILLIPLEX MAP Human Autoimmune Autoantibody Panel (HAIAB-10K, MilliporeSigma, at a 1:50 dilution for GaP donors and 1:200 dilution for SLE donors) on the Luminex xMAP system. In all cases (ELISA, TruePLEX, and MILLIPLEX), background signals were subtracted before plotting.

Flow cytometry. PBMCs were washed and blocked in PBS supplemented with Fc blocker (422302, BioLegend) for 15 minutes and then stained with antibodies against surface makers for 1 hour (BioLegend: CD303-BV421 354212, CD123-BV510 306022, CD14-PE 301806, CD16-APC Cy7 360710, CD40-PE 334308, CD45-PerCP 304026, CD38-BV711 303528, IgD-APC 348222, CD27-BV421 356418, CD19BV510 302242, CD20-BV711 302342, PE secondary 406421; BD Biosciences: CD24-PerCP-Cy5.5 561647; Abcam: cit-H3 ab5103; Novus: MPO-PerCP NB100-64803PCP; eBioscience: CD66b-APC 1706662; 
Molecular Probes, Thermo Fisher Scientific: LIVE/DEAD fixable green stain L23101). After staining, cells were washed 2 times in PBS without $\mathrm{Mg}^{2+}$ or $\mathrm{Ca}^{2+}$ and then fixed in $2 \%$ paraformaldehyde (PFA) before analysis on a BD Fortessa or BD LSR flow cytometer. For intracellular IRF5 staining, after overnight fixation, cells were permeabilized the following day in $0.1 \%$ Triton X-100, rinsed in PBS twice before blocking in 2\% BSA solution, and then stained with anti-IRF5 antibody conjugated to Alexa Fluor 488 (Abcam ab193245). B cell subsets were gated as $\mathrm{CD} 45^{+} \mathrm{CD} 19^{+}$then $\mathrm{CD} 27^{+} \mathrm{IgD}{ }^{-}$for switched memory, $\mathrm{CD} 27^{+} \mathrm{IgD}^{+}$for nonswitched memory, IgD ${ }^{+} \mathrm{CD} 27^{-} \mathrm{CD} 24^{-} \mathrm{CD} 38^{-}$for naive, $\mathrm{IgD}^{+} \mathrm{CD} 27^{-} \mathrm{CD} 24^{\mathrm{hi}} \mathrm{CD} 38^{\mathrm{hi}}$ for transitional, and $\mathrm{IgD}^{-} \mathrm{CD} 38^{+}$for plasma cells. Monocyte subsets were gated as $\mathrm{CD} 45^{+} \mathrm{CD} 14^{+} \mathrm{CD} 16^{-}$for classical, $\mathrm{CD} 45^{+} \mathrm{CD} 14^{+} \mathrm{CD} 16^{+}$for intermediate, and $\mathrm{CD} 45^{+} \mathrm{CD} 14^{\text {lo }} \mathrm{CD} 16^{\text {lo }}$ for inflammatory. $\mathrm{pDCs}$ were gated as $\mathrm{CD} 45^{+} \mathrm{CD} 123^{+} \mathrm{BDCA} 2^{+}$. NETs were gated as $\mathrm{CD}_{6} 6 \mathrm{~b}^{+} \mathrm{cit}-\mathrm{H} 3^{+} \mathrm{MPO}^{+}$. The LIVE/DEAD viability discrimination dye was used primarily on ex vivo cocultures (13).

Imaging flow cytometry. Imaging flow was performed as previously described on an Amnis ImageStream ${ }^{\mathrm{X}}$ Mark II Imaging Flow Cytometer (MilliporeSigma) $(13,24)$. Briefly, freshly isolated PBMCs were either left unstimulated or stimulated with R848 (500 ng/mL) for 2 hours, followed by surface staining with the following markers: anti-CD14-PE, 301806; anti-CD19-BV510, 302242; anti-CD24-PE, 311106; anti-CD123BV510, 306014; anti-BDCA2-BV421, 354212 (from BioLegend), and anti-CD45-APC/Cy7, 557833; anti-CD16-PerCP/Cy5.5, 557758; anti-IgD-APC, 555778; and anti-CD38-PE-CF594, 562288 (from $\mathrm{BD}$ Biosciences). After surface staining, PBMCs were fixed overnight in 1\% PFA. In some instances, cells were permeabilized $(13,24)$ and blocked in 5\% BSA solution before staining for intracellular IRF5 (Abcam ab193245) or IFN- $\alpha$ (Miltenyi Biotec 130-099-098). Prior to acquisition, the nuclear dye DRAQ5 (Thermo Fisher Scientific, 62251) was added at a 1:500 dilution. Images were acquired on the ImageStream ${ }^{\mathrm{X}}$ using the $\times 40$ objective. Raw image files were acquired with INSPIRE software (Luminex Corporation). A compensation matrix was used for acquisition and then applied to acquired data to correct for spectral overlap followed by data analysis using IDEAS 5.0 software (Luminex Corporation). Similar gating strategies as those for traditional flow cytometry were used $(13,24)$. Briefly, cells were first filtered through the bright-field area versus bright-field aspect ratio gate to exclude nonviable and doublet events. Following that a similar gate of the DRAQ5 nuclear channel was used. This added an extra measure of stringency for cell viability. $\mathrm{B}$ cell subsets were gated as $\mathrm{CD} 45^{+} \mathrm{CD} 19^{+}$and then $\mathrm{IgD}^{+} \mathrm{CD} 38^{-}$for naive, $\mathrm{CD} 24^{+} \mathrm{CD} 38^{+}$for transitional, and $\mathrm{IgD}^{-} \mathrm{CD} 38^{+}$for plasma cells. Monocyte subsets were gated as $\mathrm{CD} 45^{+} \mathrm{CD} 14^{+}$and then $\mathrm{CD} 14^{+} \mathrm{CD} 16^{-}$for classical, $\mathrm{CD} 14^{+} \mathrm{CD} 16^{+}$for intermediate, and $\mathrm{CD} 14^{-} \mathrm{CD} 16^{+}$for inflammatory. pDCs were gated as $\mathrm{CD} 45^{+} \mathrm{C}-$ D123 ${ }^{+}$BDCA2 ${ }^{+}$. To measure IRF5 activation, a gate on Gradient RMS for DRAQ5 channel was added to exclude out-of-focus events, and IRF5 nuclear translocation was determined through use of the similarity score feature contrasting IRF5 staining with DRAQ5 staining (Supplemental Figure 3). A similarity score of 3 or above was considered a translocation event.

In vitro plasma cell differentiation. PBMCs were diluted to a concentration of $5 \times 10^{7}$ cells $/ \mathrm{mL}$ and naive B cells isolated using a STEMCELL Technologies kit (EasySep, 19254). Magnetic separation was performed to achieve a greater than $95 \%$ enriched population of naive $\mathrm{B}$ cells $\left(\mathrm{CD} 19^{+} \mathrm{CD}_{20}{ }^{+} \operatorname{IgD} \mathrm{CD}^{+} \mathrm{CD}^{-}\right)$, as determined by flow cytometry (13). Isolated naive B cells were then cultured in 96-well U-bottom plates at a minimum density of $1 \times 10^{6}$ with $150 \mathrm{ng} / \mathrm{mL}$ CD40L (Peprotech 310-02) alone or with $100 \mathrm{ng} / \mathrm{mL}$ IL-21 (Peprotech 200-21), $10 \mu \mathrm{g} / \mathrm{mL}$ anti-IgM antibody (Southern Biotech 2020-01), and $2.5 \mu \mathrm{g} / \mathrm{mL}$ CpG-B (Hycult Tech HC4039) for 7 days.

Immunofluorescence analysis of NETs. To assess spontaneous NETosis, neutrophils were incubated at $37^{\circ} \mathrm{C}$ for 4 hours for flow cytometry and simultaneously plated on poly-L-lysine-coated coverslips for 4 hours for immunocytochemistry (ICC). For flow cytometric analysis, cells were stained with CD66b-APC, cit-H3 primary antibody, and MPO-PerCP followed by PE secondary antibody. For ICC, cells were washed with PBS once and fixed with $4 \%$ formalin for 5 minutes. Cells were then permeabilized using $0.1 \%$ Triton X-100 for 10 minutes followed by blocking with 3\% BSA for 2 hours. After washing, cells were stained overnight with primary anti-rabbit cit-H3 (Abcam ab5103) and anti-mouse MPO (Abcam ab25989) antibodies. After overnight incubation, cells were washed, blocked for 1 hour, and then stained with goat anti-rabbit Cy3 (Jackson ImmunoResearch 711165152) and goat anti-mouse Alexa Fluor 488 (Invitrogen, Thermo Fisher Scientific, A21042). Another set of neutrophils plated on poly-L-lysine-coated coverslips were stained directly with $5 \mu \mathrm{M}$ Sytox green (Molecular Probes, Thermo Fisher Scientific, S7020) and DAPI (BioLegend 422801) to examine NETs. Treatment of neutrophils with $25 \mathrm{nM}$ PMA for 4 hours was used as a positive control to induce NETs. Cells were washed and analyzed using the EVOS cell imaging system (Thermo Fisher Scientific). 
Cell sorting and $q P C R$. PBMCs were labeled with anti-CD45, -CD14, and -CD16 fluorescent-conjugated antibodies. $\mathrm{CD} 14^{+} \mathrm{CD} 16^{\text {lo }}$ classical monocytes and $\mathrm{CD} 14^{\mathrm{lo}} \mathrm{CD} 16^{+}$inflammatory monocytes were sorted on a BD FACSAria. RNA was prepared from sorted cells by Trizol isolation, followed by qPCR with primers for IL8 (5'-ACTGAGAGTGATTGAGAGTGGAC, 3'-AACCCTCTGCACCCAGTTTTC). Primers for qPCR of IRF5 were previously described (84). Each sample was assayed in replicates of 3 . Threshold values $(\mathrm{Ct})$ were averaged over each sample replicate, followed by normalization via the $\Delta \Delta \mathrm{Ct}$ method to the $\beta$-actin housekeeping gene (13).

Ex vivo coculture. Purified neutrophils $\left(4 \times 10^{6} / 200 \mu \mathrm{L}\right)$ were plated on poly-L-lysine-coated coverslips in a 24-well plate for 2 hours. Nonadherent neutrophils were removed, and isolated PBMCs $\left(4 \times 10^{6} / \mathrm{mL}\right)$ were added to the wells. PBMCs were collected at 4 hours, after overnight coculture, and after 7 days of coculture to study IRF5 activation, pDC activation and expansion, and PB differentiation, respectively.

RNA-Seq sample preparation, data collection, and processing. Granulocytes were washed 3 times with RNAse-free PBS and then lysed in RLT lysis buffer with $\beta$-mercaptoethanol, as recommended by manufacturers of the QIAGEN RNeasy Mini Kit. Lysed samples were then incubated at $4^{\circ} \mathrm{C}$ for 20 minutes before further processing. Libraries were prepared and run on an Illumina HiSeq2500. Collection and data processing for counts and quality metrics were performed as previously described (85). Libraries that had fewer than 5 million total reads, a median coefficient of variation of coverage greater than $50 \%$, or less than $75 \%$ alignment to the reference genome were excluded. An additional library was excluded due to outlying quality variance identified by principal components analysis (PCA). Following quality control filtering, 11 of 14 samples ( 5 risk and 6 nonrisk) remained for downstream analysis. Counts were normalized using trimmed mean of $\mathrm{M}$ values (TMM) $(86,87)$. Genes were included for downstream analysis if they had at least 1 count per million in $10 \%$ of the libraries.

$R N A$-Seq data analysis. All RNA-Seq analyses were performed in R using TMM-normalized, $\log _{2}$-transformed counts. Limma-voom was used for differential gene expression analysis (88). The most variable quality metric identified by PCA (number of mapped reads with duplicates) was included in the model as a covariate to control for quality-related variance. Gene set statistical testing was performed using roast, a rotation gene set analysis function in the limma $\mathrm{R}$ package. Gene set enrichment was visualized using the barcodeplot function. Relative gene expression was calculated as per-gene $Z$ scores across all samples, and the mean $Z$ score was calculated for each gene per risk group.

Data availability. RNA-Seq data will be deposited into GEO, accession GSE137067, on publication.

Statistics. Data presented are from single rounds of matched blood draws consisting of $n=11-14$ homozygous nonrisk and $n=11-12$ homozygous risk donors. These data are representative of 4 or more independent blood draws over a 4-year period. GraphPad Prism 5 was used for statistical analysis and graphing. Prior to testing, graph kurtosis was analyzed to determine normal distribution. A 2-tailed $t$ test was used for comparisons between 2 samples with normal distribution. Mann-Whitney $U$ test was used to compare 2 samples without normal distribution. For comparisons of 1 factor over multiple groups, a 1-way ANOVA was performed with Tukey's or Bonferroni's post hoc test for significance. $P<0.05$ was considered statistically significant.

Study approval. All experiments were approved by The Feinstein Institutes for Medical Research IRB. Informed consent was obtained from all healthy donors prior to inclusion in the study, and experiments were performed in accordance with institutional and regional guidelines.

\section{Author contributions}

$\mathrm{DL}$ and BM equally contributed to the immune phenotyping of GaP donors. SS performed ANA analysis, and VN performed qPCR analysis. KRS and PKG performed haplotype analysis. KD and PL performed RNA-Seq and bioinformatics analysis. BJB designed the research and supervised the project. DL, BM, KRS, PKG, and BJB wrote the manuscript.

\section{Acknowledgments}

We thank members of the GaP Registry and G. Klein, M. DeFranco, and K.M. Elmaliki for consenting and scheduling donors. This work was supported by grants from the Alliance for Lupus Research, NIH's National Institute of Arthritis and Musculoskeletal and Skin Diseases AR065959-01, Department of Defense CDMRP LRP W81XWH-18-1-0674, Flight Attendant Medical Research Institute, and Lupus Research Alliance to BJB. 
Address correspondence to: Betsy J. Barnes, The Feinstein Institute for Medical Research, 350 Community Drive, Manhasset, New York 11030, USA. Phone: 516.562.0434; Email: bbarnes1@northwell.edu.

1. Arbuckle MR, et al. Development of autoantibodies before the clinical onset of systemic lupus erythematosus. $N$ Engl J Med. 2003;349(16):1526-1533.

2. Heinlen LD, et al. Ribosomal P autoantibodies are present before SLE onset and are directed against non-C-terminal peptides. J Mol Med. 2010;88(7):719-727.

3. Olsen NJ, Karp DR. Autoantibodies and SLE: the threshold for disease. Nat Rev Rheumatol. 2014;10(3):181-186.

4. Odendahl M, et al. Disturbed peripheral B lymphocyte homeostasis in systemic lupus erythematosus. J Immunol. 2000;165(10):5970-5979.

5. Tipton CM, et al. Diversity, cellular origin and autoreactivity of antibody-secreting cell population expansions in acute systemic lupus erythematosus. Nat Immunol. 2015;16(7):755-765.

6. Bennett L, et al. Interferon and granulopoiesis signatures in systemic lupus erythematosus blood. J Exp Med. 2003;197(6):711-723.

7. Chaussabel $\mathrm{D}$, et al. A modular analysis framework for blood genomics studies: application to systemic lupus erythematosus. Immunity. 2008;29(1):150-164.

8. Chiche L, et al. Modular transcriptional repertoire analyses of adults with systemic lupus erythematosus reveal distinct type I and type II interferon signatures. Arthritis Rheumatol. 2014;66(6):1583-1595.

9. Streicher K, et al. The plasma cell signature in autoimmune disease. Arthritis Rheumatol. 2014;66(1):173-184.

10. Eames HL, Corbin AL, Udalova IA. Interferon regulatory factor 5 in human autoimmunity and murine models of autoimmune disease. Transl Res. 2016;167(1):167-182.

11. Takaoka A, et al. Integral role of IRF-5 in the gene induction programme activated by Toll-like receptors. Nature. 2005;434(7030):243-249.

12. Schoenemeyer A, et al. The interferon regulatory factor, IRF5, is a central mediator of toll-like receptor 7 signaling. J Biol Chem. 2005;280(17):17005-17012.

13. De S, et al. B cell-intrinsic role for IRF5 in TLR9/BCR-induced human B cell activation, proliferation, and plasmablast differentiation. Front Immunol. 2017;8:1938.

14. Sigurdsson S, et al. Polymorphisms in the tyrosine kinase 2 and interferon regulatory factor 5 genes are associated with systemic lupus erythematosus. Am J Hum Genet. 2005;76(3):528-537.

15. Sigurdsson S, et al. Comprehensive evaluation of the genetic variants of interferon regulatory factor 5 (IRF5) reveals a novel 5 bp length polymorphism as strong risk factor for systemic lupus erythematosus. Hum Mol Genet. 2008;17(6):872-881.

16. Graham RR, et al. A common haplotype of interferon regulatory factor 5 (IRF5) regulates splicing and expression and is associated with increased risk of systemic lupus erythematosus. Nat Genet. 2006;38(5):550-555.

17. Graham RR, et al. Three functional variants of IFN regulatory factor 5 (IRF5) define risk and protective haplotypes for human lupus. Proc Natl Acad Sci USA. 2007;104(16):6758-6763.

18. Löfgren SE, et al. Promoter insertion/deletion in the IRF5 gene is highly associated with susceptibility to systemic lupus erythematosus in distinct populations, but exerts a modest effect on gene expression in peripheral blood mononuclear cells. J Rheumatol. 2010;37(3):574-578.

19. Cunninghame Graham DS, et al. Association of IRF5 in UK SLE families identifies a variant involved in polyadenylation. Hum Mol Genet. 2007;16(6):579-591.

20. Feng D, et al. Genetic variants and disease-associated factors contribute to enhanced interferon regulatory factor 5 expression in blood cells of patients with systemic lupus erythematosus. Arthritis Rheum. 2010;62(2):562-573.

21. Niewold TB, Kelly JA, Flesch MH, Espinoza LR, Harley JB, Crow MK. Association of the IRF5 risk haplotype with high serum interferon-alpha activity in systemic lupus erythematosus patients. Arthritis Rheum. 2008;58(8):2481-2487.

22. Niewold TB, et al. IRF5 haplotypes demonstrate diverse serological associations which predict serum interferon alpha activity and explain the majority of the genetic association with systemic lupus erythematosus. Ann Rheum Dis. 2012;71(3):463-468.

23. Mancl ME, et al. Two discrete promoters regulate the alternatively spliced human interferon regulatory factor-5 isoforms. Multiple isoforms with distinct cell type-specific expression, localization, regulation, and function. J Biol Chem. 2005;280(22):21078-21090.

24. Stone RC, et al. Interferon regulatory factor 5 activation in monocytes of systemic lupus erythematosus patients is triggered by circulating autoantigens independent of type I interferons. Arthritis Rheum. 2012;64(3):788-798.

25. Barnes BJ. Genetic versus non-genetic drivers of SLE: implications of IRF5 dysregulation in both roads leading to SLE. Curr Rheumatol Rep. 2019;21(1):2.

26. Gregersen PK, et al. The Genotype and Phenotype $(\mathrm{GaP})$ registry: a living biobank for the analysis of quantitative traits. Immunol Res. 2015;63(1-3):107-112.

27. Cherian TS, Kariuki SN, Franek BS, Buyon JP, Clancy RM, Niewold TB. Brief Report: IRF5 systemic lupus erythematosus risk haplotype is associated with asymptomatic serologic autoimmunity and progression to clinical autoimmunity in mothers of children with neonatal lupus. Arthritis Rheum. 2012;64(10):3383-3387.

28. Berggren O, et al. IFN- $\alpha$ production by plasmacytoid dendritic cell associations with polymorphisms in gene loci related to autoimmune and inflammatory diseases. Hum Mol Genet. 2015;24(12):3571-3581.

29. Stone RC, et al. RNA-Seq for enrichment and analysis of IRF5 transcript expression in SLE. PLoS ONE. 2013;8(1):e54487.

30. Matta B, Barnes BJ. Coordination between innate immune cells, type I IFNs and IRF5 drives SLE pathogenesis [published online ahead of print May 23, 2019]. Cytokine. doi:10.1016/j.cyto.2019.05.018.

31. Jego G, Palucka AK, Blanck JP, Chalouni C, Pascual V, Banchereau J. Plasmacytoid dendritic cells induce plasma cell differentiation through type I interferon and interleukin 6. Immunity. 2003;19(2):225-234.

32. Zheng N, et al. Increased abundance of plasmacytoid dendritic cells and interferon-alpha induces plasma cell differentiation in patients of IgA nephropathy. Mediators Inflamm. 2017;2017:4532409. 
33. Yu Y, Su K. Neutrophil Extracellular Traps and Systemic Lupus Erythematosus. J Clin Cell Immunol. 2013;4:139.

34. Lande R, et al. Neutrophils activate plasmacytoid dendritic cells by releasing self-DNA-peptide complexes in systemic lupus erythematosus. Sci Transl Med. 2011;3(73):73ra19.

35. Garcia-Romo GS, et al. Netting neutrophils are major inducers of type I IFN production in pediatric systemic lupus erythematosus. Sci Transl Med. 2011;3(73):73ra20.

36. Hakkim A, et al. Impairment of neutrophil extracellular trap degradation is associated with lupus nephritis. Proc Natl Acad Sci USA. 2010;107(21):9813-9818.

37. Leffler J, et al. Neutrophil extracellular traps that are not degraded in systemic lupus erythematosus activate complement exacerbating the disease. J Immunol. 2012;188(7):3522-3531.

38. Kaplan MJ, Radic M. Neutrophil extracellular traps: double-edged swords of innate immunity. J Immunol. 2012;189(6):2689-2695

39. Yang H, Biermann MH, Brauner JM, Liu Y, Zhao Y, Herrmann M. New insights into neutrophil extracellular traps: mechanisms of formation and role in inflammation. Front Immunol. 2016;7:302.

40. Arango Duque G, Descoteaux A. Macrophage cytokines: involvement in immunity and infectious diseases. Front Immunol. 2014;5:491.

41. Praefcke GJK. Regulation of innate immune functions by guanylate-binding proteins. Int J Med Microbiol. 2018;308(1):237-245

42. Finethy R, et al. Guanylate binding proteins enable rapid activation of canonical and noncanonical inflammasomes in Chlamydia-infected macrophages. Infect Immun. 2015;83(12):4740-4749.

43. Pilla DM, et al. Guanylate binding proteins promote caspase-11-dependent pyroptosis in response to cytoplasmic LPS. Proc Natl Acad Sci USA. 2014;111(16):6046-6051.

44. Meunier E, et al. Caspase-11 activation requires lysis of pathogen-containing vacuoles by IFN-induced GTPases. Nature 2014;509(7500):366-370.

45. Wallet $P$, et al. IFN- $\gamma$ extends the immune functions of Guanylate Binding Proteins to inflammasome-independent antibacterial activities during Francisella novicida infection. PLoS Pathog. 2017;13(10):e1006630.

46. Man SM, Place DE, Kuriakose T, Kanneganti TD. Interferon-inducible guanylate-binding proteins at the interface of cell-autonomous immunity and inflammasome activation. J Leukoc Biol. 2017;101(1):143-150.

47. Broz P, Dixit VM. Inflammasomes: mechanism of assembly, regulation and signalling. Nat Rev Immunol. 2016;16(7):407-420.

48. Martinelli S, et al. Induction of genes mediating interferon-dependent extracellular trap formation during neutrophil differentiation. J Biol Chem. 2004;279(42):44123-44132.

49. Branzk N, Papayannopoulos V. Molecular mechanisms regulating NETosis in infection and disease. Semin Immunopathol. 2013;35(4):513-530.

50. Mahajan A, Herrmann M, Muñoz LE. Clearance deficiency and cell death pathways: a model for the pathogenesis of SLE. Front Immunol. 2016;7:35.

51. Elloumi N, et al. Differential reactive oxygen species production of neutrophils and their oxidative damage in patients with active and inactive systemic lupus erythematosus. Immunol Lett. 2017;184:1-6.

52. Perazzio SF, Salomão R, Silva NP, Andrade LE. Increased neutrophil oxidative burst metabolism in systemic lupus erythematosus. Lupus. 2012;21(14):1543-1551.

53. Bengtsson AA, et al. Low production of reactive oxygen species in granulocytes is associated with organ damage in systemic lupus erythematosus. Arthritis Res Ther. 2014;16(3):R120.

54. Marzocchi-Machado CM, Alves CM, Azzolini AE, Polizello AC, Carvalho IF, Lucisano-Valim YM. Fcgamma and complement receptors: expression, role and co-operation in mediating the oxidative burst and degranulation of neutrophils of Brazilian systemic lupus erythematosus patients. Lupus. 2002;11(4):240-248.

55. Hultqvist M, Olsson LM, Gelderman KA, Holmdahl R. The protective role of ROS in autoimmune disease. Trends Immunol. 2009;30(5):201-208.

56. Olsson LM, et al. Copy number variation of the gene NCF1 is associated with rheumatoid arthritis. Antioxid Redox Signal. 2012;16(1):71-78.

57. Campbell AM, Kashgarian M, Shlomchik MJ. NADPH oxidase inhibits the pathogenesis of systemic lupus erythematosus. Sci Transl Med. 2012;4(157):157ra141.

58. Kelkka T, et al. Reactive oxygen species deficiency induces autoimmunity with type 1 interferon signature. Antioxid Redox Signal. 2014;21(16):2231-2245.

59. Jacob CO, et al. Lupus-associated causal mutation in neutrophil cytosolic factor 2 (NCF2) brings unique insights to the structure and function of NADPH oxidase. Proc Natl Acad Sci USA. 2012;109(2):E59-E67.

60. Kienhöfer D, et al. Experimental lupus is aggravated in mouse strains with impaired induction of neutrophil extracellular traps. JCI Insight. 2017;2(10):92920.

61. Calise J, Marquez Renteria S, Gregersen PK, Diamond B. Lineage-specific functionality of an interferon regulatory factor 5 lupus risk haplotype: lack of B cell intrinsic effects. Front Immunol. 2018;9:996.

62. Hedl M, Abraham C. IRF5 risk polymorphisms contribute to interindividual variance in pattern recognition receptor-mediated cytokine secretion in human monocyte-derived cells. J Immunol. 2012;188(11):5348-5356.

63. Hed1 M, Yan J, Abraham C. IRF5 and IRF5 disease-risk variants increase glycolysis and human M1 macrophage polarization by regulating proximal signaling and Akt2 activation. Cell Rep. 2016;16(9):2442-2455.

64. Lin R, Yang L, Arguello M, Penafuerte C, Hiscott J. A CRM1-dependent nuclear export pathway is involved in the regulation of IRF-5 subcellular localization. J Biol Chem. 2005;280(4):3088-3095.

65. Cheng TF, Brzostek S, Ando O, Van Scoy S, Kumar KP, Reich NC. Differential activation of IFN regulatory factor (IRF)-3 and IRF-5 transcription factors during viral infection. J Immunol. 2006;176(12):7462-7470.

66. Lu R, et al. Dysregulation of innate and adaptive serum mediators precedes systemic lupus erythematosus classification and improves prognostic accuracy of autoantibodies. J Autoimmun. 2016;74:182-193.

67. Gilliet M, Cao W, Liu YJ. Plasmacytoid dendritic cells: sensing nucleic acids in viral infection and autoimmune diseases. Nat Rev Immunol. 2008;8(8):594-606.

68. Hoffmann HH, Schneider WM, Rice CM. Interferons and viruses: an evolutionary arms race of molecular interactions. Trends 
Immunol. 2015;36(3):124-138.

69. Swiecki M, Colonna M. The multifaceted biology of plasmacytoid dendritic cells. Nat Rev Immunol. 2015;15(8):471-485.

70. McKenna K, Beignon AS, Bhardwaj N. Plasmacytoid dendritic cells: linking innate and adaptive immunity. $J$ Virol. 2005;79(1):17-27.

71. Poeck H, et al. Plasmacytoid dendritic cells, antigen, and CpG-C license human B cells for plasma cell differentiation and immunoglobulin production in the absence of T-cell help. Blood. 2004;103(8):3058-3064

72. Menon M, Blair PA, Isenberg DA, Mauri C. A regulatory feedback between plasmacytoid dendritic cells and regulatory b cells is aberrant in systemic lupus erythematosus. Immunity. 2016;44(3):683-697.

73. Smith CK, Kaplan MJ. The role of neutrophils in the pathogenesis of systemic lupus erythematosus. Curr Opin Rheumatol. 2015;27(5):448-453

74. Farkas L, Beiske K, Lund-Johansen F, Brandtzaeg P, Jahnsen FL. Plasmacytoid dendritic cells (natural interferon- alpha/ beta-producing cells) accumulate in cutaneous lupus erythematosus lesions. Am J Pathol. 2001;159(1):237-243.

75. Crispín JC, Vargas-Rojas MI, Monsiváis-Urenda A, Alcocer-Varela J. Phenotype and function of dendritic cells of patients with systemic lupus erythematosus. Clin Immunol. 2012;143(1):45-50.

76. Migita K, et al. Reduced blood BDCA-2+ (lymphoid) and CD11c+ (myeloid) dendritic cells in systemic lupus erythematosus. Clin Exp Immunol. 2005;142(1):84-91.

77. Khan SA, et al. Active systemic lupus erythematosus is associated with decreased blood conventional dendritic cells. Exp Mol Pathol. 2013;95(2):121-123.

78. Macal M, et al. Self-renewal and Toll-like receptor signaling sustain exhausted plasmacytoid dendritic cells during chronic viral infection. Immunity. 2018;48(4):730-744.e5.

79. Feldman S, et al. Decreased interferon-alpha production in HIV-infected patients correlates with numerical and functional deficiencies in circulating type 2 dendritic cell precursors. Clin Immunol. 2001;101(2):201-210.

80. Duan XZ, Wang M, Li HW, Zhuang H, Xu D, Wang FS. Decreased frequency and function of circulating plasmocytoid dendritic cells (pDC) in hepatitis B virus infected humans. J Clin Immunol. 2004;24(6):637-646.

81. Zhan Y, et al. Plasmacytoid dendritic cells are short-lived: reappraising the influence of migration, genetic factors and activation on estimation of lifespan. Sci Rep. 2016;6:25060.

82. Zuniga EI, Macal M, Lewis GM, Harker JA. Innate and adaptive immune regulation during chronic viral infections. Annu Rev Virol. 2015;2(1):573-597.

83. Machiela MJ, Chanock SJ. LDlink: a web-based application for exploring population-specific haplotype structure and linking correlated alleles of possible functional variants. Bioinformatics. 2015;31(21):3555-3557.

84. Cevik O, et al. Interferon regulatory factor 5 (IRF5) suppresses hepatitis $\mathrm{C}$ virus (HCV) replication and HCV-associated hepatocellular carcinoma. J Biol Chem. 2017;292(52):21676-21689.

85. Linsley PS, Greenbaum CJ, Rosasco M, Presnell S, Herold KC, Dufort MJ. Elevated T cell levels in peripheral blood predict poor clinical response following rituximab treatment in new-onset type 1 diabetes. Genes Immun. 2019;20(4):293-307.

86. Robinson MD, McCarthy DJ, Smyth GK. edgeR: a Bioconductor package for differential expression analysis of digital gene expression data. Bioinformatics. 2010;26(1):139-140

87. Robinson MD, Oshlack A. A scaling normalization method for differential expression analysis of RNA-seq data. Genome Biol. 2010;11(3):R25

88. Ritchie ME, et al. limma powers differential expression analyses for RNA-sequencing and microarray studies. Nucleic Acids Res. 2015;43(7):e47. 